\title{
The Composition and Antioxidant Activity of Bound Phenolics in Three Legumes, and Their Metabolism and Bioaccessibility of Gastrointestinal Tract
}

\author{
Liuying Zhu ${ }^{1}$, Wenting $\mathrm{Li}^{1}$, Zeyuan Deng ${ }^{1,2}$, Hongyan $\mathrm{Li}^{1,2}$ and Bing Zhang ${ }^{1, *}$ \\ 1 State Key Laboratory of Food Science and Technology, Nanchang University, \\ Nanchang 330047, Jiangxi, China; 402337519034@email.ncu.edu.cn (L.Z.); \\ 407213315069@email.ncu.edu.cn (W.L.); dengzy@ncu.edu.cn (Z.D.); lihongyan@ncu.edu.cn (H.L.) \\ 2 Institute for Advanced Study, University of Nanchang, Nanchang 330031, Jiangxi, China \\ * Correspondence: zhangbingair@ncu.edu.cn; Tel./Fax: +86-7918-830-4449
}

Received: 11 November 2020; Accepted: 4 December 2020; Published: 7 December 2020

\begin{abstract}
The composition and antioxidant activity of bound phenolics in three legumes (soybean, vicia faba, and kidney bean), and their metabolism and bioaccessibility in the gastrointestinal tract were investigated in this study. The total phenolic content, total flavonoid content, and antioxidant activities (ABTS and FRAP) were evaluated. The phytochemical compositions of the three legumes after acid/alkaline hydrolysis, simulated gastrointestinal digestion, and colonic fermentation were identified and quantified by UPLC-ESI-QTOF-MS/MS and HPLC-ESI-QqQ-MS/MS. The results showed that the three legumes were rich in bound phenolic compounds, and possessed a strong antioxidant activity; among which kidney bean showed a higher bound flavonoid content and antioxidant activity than the other two legumes. Alkaline hydrolysis allowed a more thorough extraction of the bound phenolics of the three legumes than acid hydrolysis. The released contents of bound phenolics were extremely low in in vitro digestion, whereas colonic fermentation favored the release of more phenolic compounds. Kidney bean, which presented the highest bound flavonoid content and antioxidant activity, had the lowest bioaccessibility. Our study provides a wider insight into the constituents and bioavailability of bound phenolic compounds in the three legumes.
\end{abstract}

Keywords: soybean; vicia faba; kidney bean; acid/alkaline hydrolysis; in vitro digestion; colonic fermentation

\section{Introduction}

Plant polyphenols are a kind of secondary metabolite, which are widely found in various foods, such as legumes, fruits, vegetables, herbs, and cereals [1]. They are important bioactive components in our diet. Many studies associated polyphenol-rich diets with several health effects in human, and triggered the increasing interest in polyphenol. The phenolics present in plants mainly include two parts, soluble phenols that can be extracted by organic solvents, and insoluble bound phenols that are not extracted by organic solvents [2]; the bound phenolic mainly bonds with cellulose, hemicellulose, pectin, protein, and arabinoxylans by ester and $\mathrm{C}-\mathrm{C}$ bonds in cell walls, acting as building materials for the cell wall matrix, so they cannot be extracted by the organic solvent and present in the residue [3,4]. Nevertheless, the chemical bonds between the substances can be destroyed by acid hydrolysis, alkaline hydrolysis, or enzymatic hydrolysis [5], thus releasing bound phenolic substances. At present, most research on phenolic substances are concentrated on the soluble phenols, while research on bound phenolic has been neglected, resulting in the underestimation of the total content of plant polyphenols, their antioxidant capacity, and their effects on the human body. 
In recent years, legumes as a human diet, have been winning growing interest for their health effects, and are recommended as great source of bioactive phenolic compounds, which constitute plenty of biological functions [6]. Several studies have demonstrated that legumes possess numerous bioactive properties, which have been largely connected with their phenolic compounds. García-Lafuente et al. [7] showed that phenolic extract of common bean can stimulate the expression of cytokine mRNA in macrophages, and reduce the production of nitrous oxide, indicating that it has high antioxidant and anti-inflammatory activities. It has been reported that there are abundant phenolic acids (delphinidin and ferulic acid) and anthocyanins in beans, which are usually applied as functional food ingredients, and may be beneficial to health, such as being anti-tumor, and prevention of cardiovascular disease [8]. Additionally, phenolic compounds in legumes can control postprandial glucose response by inhibiting $\alpha$-glucosidase to reduce the digestion and absorption of glucose in the intestine, suggesting a use for the management of type 2 diabetes mellitus [9]. In short, these results clarify the bioaccessibility of the phenolic compounds in legumes.

As widely known, the health benefits of phenolic compounds are closely related to metabolism [10]. Mosele et al. [11] reported the absorption of soluble polyphenols mainly in the small intestine, while bound polyphenols are metabolized by the intestinal flora in the colon, instead of being absorbed in the small intestine [12]. Therefore, it is of great significance to study the chemical transformation of bound polyphenols during gastrointestinal digestion and colonic fermentation. The multiple advantages of in vitro research, such as being fast and cheap, better condition control, and less ethical restrictions [13], have made it an alternative method to studying the metabolic process of biologically active substances in vivo.

Although legumes are considered a potential source of soluble and bound phenols, there is no information on the bioaccessibility of bound phenols during gastrointestinal digestion and subsequent colonic fermentation. Soybean, vicia faba, and kidney bean are common edible beans, and rich in phenolic components [6]. Hence the present study screened these three legumes to examine their bound phenolic composition and antioxidant activities, as well as their metabolism and bioaccessibility of bound phenolics by in vitro gastrointestinal digestion and colon fermentation (anaerobically incubated with human feces).

\section{Materials and Methods}

\subsection{Materials}

Soybean (Glycine max (Linn.) Merr.), vicia faba (Vicia faba L.), and kidney bean (Phaseolus vulgaris L.) were obtained from the local supermarket in Nanchang, Jiangxi, China (February 2016). The three legumes were freeze-dried, milled to a fine powder with a high-speed universal pulverizer and sieved through 20 meshes, then kept in a sealed bag at $-80^{\circ} \mathrm{C}$ until extracted.

\subsection{Chemicals and Reagents}

P-hydroxybenzoic acid, p-coumaric acid, chlorogenic acid, ferulic acid, procatechuic acid, gallic acid, sinapic acid, catechin, daidzein, daidzin, quercetin, hyperoside, genistein, genistin, rutin, naringenin, glycitein, glycitin, biochanin $\mathrm{A}$, and vitexin as phenolic standards were bought from Aladdin (Shanghai, China). $\mathrm{Na}_{2} \mathrm{CO}_{3}, \mathrm{FeCl}_{3} \cdot 6 \mathrm{H}_{2} \mathrm{O}, \mathrm{CaCl}_{2}, \mathrm{NaCl}, \mathrm{KCl}, \mathrm{KH}_{2} \mathrm{PO}_{4}$, and $\mathrm{K}_{2} \mathrm{HPO}_{4}$, $\mathrm{NaHCO}_{3}, \mathrm{MgSO}_{4} \mathrm{H}_{2} \mathrm{O}$, L-cysteine, $\mathrm{K}_{2} \mathrm{~S}_{2} \mathrm{O}_{8}, \mathrm{FeSO}_{4}, \mathrm{HCl}$, and $\mathrm{NaOH}$ were obtained from Xilong (Guangzhou, China). Acetate buffer, PBS buffer, Tween resazurin solution, arabinogalactan, xylan, $\alpha$-amylase, trypsin, bile salt, Trolox, Folin-Ciocalteu, 2,2' -azinobis-(3-ethylbenzthiazoline-6-sulphonate) (ABTS), and 1,3,5-tri(2-pyridyl)-2,4,6-triazine (TPTZ) were bought from Sigma (St. Louis, MO, USA). HPLC-grade solvents, including methanol and formic acid, were obtained from Merck (Darmstadt, Germany). The water used in this work was ultrapure water produced by a Milli-Q system. 


\subsection{Extraction of Bound Phenolic Compounds}

The samples $(1 \mathrm{~g})$ of the three legumes were weighed in a $50 \mathrm{~mL}$ centrifuge tube to extract free phenolic compounds in an ultrasonic bath at room temperature with $15 \mathrm{~mL} \mathrm{80 \%}(v / v)$ aqueous methanol for $1 \mathrm{~h}$, then centrifuged $(4200 \times g)$ for $10 \mathrm{~min}$ to collect the supernatant. The samples were extracted four times, and the methanol extract was free phenolic compounds. The residues were used to extract bound phenolic compounds according to the methods of Peng Han and Kim, K.-H, with slight modifications $[14,15]$. The bound phenolics obtained by alkaline hydrolysis were hydrolyzed at room temperature with $20 \mathrm{~mL} \mathrm{NaOH}(2 \mathrm{M})$ under nitrogen for $4 \mathrm{~h}$; the $\mathrm{pH}$ of the solution was brought to 2 with $6 \mathrm{M} \mathrm{HCl}$, and then centrifuged for $10 \mathrm{~min}(4200 \times g)$ to collect the supernatant; residues were re-extracted five times. Acid hydrolysis of the samples was conducted with $25 \mathrm{~mL} \mathrm{HCl} \mathrm{(2} \mathrm{M)} \mathrm{at} 85^{\circ} \mathrm{C}$ for $1 \mathrm{~h}$; then the $\mathrm{pH}$ of the solution was adjusted to 2 using $10 \mathrm{M} \mathrm{NaOH}$ and centrifuged $(4200 \times g$, $10 \mathrm{~min}$ ) to collect the supernatant. Finally, the supernatants obtained by acid or alkaline hydrolysis were evaporated and resolved with $5 \mathrm{~mL} \mathrm{80 \%} \mathrm{methanol,} \mathrm{and} 1 \mathrm{~mL}$ was filtered through a $0.22 \mu \mathrm{m}$ PTFE membrane for HPLC analyses. The samples were preserved at $-20{ }^{\circ} \mathrm{C}$ in a refrigerator for further analysis.

\subsection{Gastrointestinal Digestion of Bound Phenolic}

The in vitro gastrointestinal (GI) digestion was conducted with the sample residues after extraction of soluble phenolic by organic solvents, and the experiment was carried out by a published method with a slightly modification [13]. First, oral digestion was simulated: $3 \mathrm{~g}$ of residue was blended with $2.1 \mathrm{~mL}$ of simulated saliva digestive fluid (SSF) electrolyte stock solution, and $0.3 \mathrm{~mL} \alpha$-amylase $(1500 \mathrm{U} / \mathrm{mL})$ and $15 \mu \mathrm{L} \mathrm{CaCl}_{2}(0.3 \mathrm{M})$ were added, then the mixture was diluted with $585 \mathrm{~mL}$ water, and the mixture oscillated at $37^{\circ} \mathrm{C}$ in a water bath for $5 \mathrm{~min}$. Second, gastric digestion was simulated: $4.5 \mathrm{~mL}$ of simulated gastric digestive fluid (SGF) electrolyte stock solution, $0.96 \mathrm{~mL}$ pepsin stock solution $(25,000 \mathrm{U} / \mathrm{mL})$, and $3 \mu \mathrm{L} \mathrm{CaCl}_{2}(0.3 \mathrm{M})$ were added to the above oral digestive system, and the $\mathrm{pH}$ of the mixture was adjusted to 3 with $0.12 \mathrm{~mL} \mathrm{HCl}(1 \mathrm{M})$; then $0.417 \mathrm{~mL}$ water was added and the solution was incubated at $37^{\circ} \mathrm{C}$ for $2 \mathrm{~h}$. Finally, intestinal digestion was simulated: $6.6 \mathrm{~mL}$ simulated intestinal digestive fluid (SIF) electrolyte stock solution, $3 \mathrm{~mL}$ trypsin $(800 \mathrm{U} / \mathrm{mL}), 1.5 \mathrm{~mL}$ fresh bile $(160 \mathrm{mM})$, and $24 \mu \mathrm{L} \mathrm{CaCl}_{2}(0.3 \mathrm{M})$ were blended in $12 \mathrm{~mL}$ gastric chime; then the mixture was regulated to $\mathrm{pH} 7.0$ with $0.15 \mathrm{~mL} \mathrm{NaOH}(1 \mathrm{M}), 1.31 \mathrm{~mL}$ water was added, and kept at $37^{\circ} \mathrm{C}$ for $2 \mathrm{~h}$, the GI digestion was centrifuged $(4200 \times g, 10 \mathrm{~min})$ to collect supernatant, and preserved at $-20^{\circ} \mathrm{C}$ until analysis.

\subsection{In Vitro Colonic Fermentation}

A previously reported method was used to conduct in vitro fermentation with some appropriate modifications [16], and the specific operation process of in vitro fermentation was undertaken as follows.

\subsubsection{In Vitro Fermentation Growth Medium Preparation}

Growth medium ( $1 \mathrm{~L}$ ) was composed of $4.5 \mathrm{~g} \mathrm{NaCl}$ and $\mathrm{KCl}, 0.5 \mathrm{~g} \mathrm{K \textrm {K } _ { 2 }} \mathrm{PO}_{4}$ and $\mathrm{K}_{2} \mathrm{HPO}_{4}, 1.5 \mathrm{~g}$ $\mathrm{NaHCO}_{3}, 0.7 \mathrm{~g} \mathrm{MgSO}_{4} \mathrm{H}_{2} \mathrm{O}, 0.8 \mathrm{~g}$ L-cysteine $\mathrm{HCl} \cdot \mathrm{H}_{2} \mathrm{O}, 0.005 \mathrm{~g} \mathrm{FeSO}_{4} \cdot 7 \mathrm{H}_{2} \mathrm{O}, 0.08 \mathrm{~g} \mathrm{CaCl}_{2}, 0.4 \mathrm{~g}$ bile salt, Tween $80(1 \mathrm{~mL})$, resazurin solution $(4 \mathrm{~mL}, 0.025 \%, w / v), 2 \mathrm{~g}$ arabinogalactan, and $1 \mathrm{~g}$ xylan. Before preparing the sample, the prepared medium was sterilized in glass vessels at $121^{\circ} \mathrm{C}$ for $15 \mathrm{~min}$.

\subsubsection{Fecal Extract Preparation}

The residues collected from the in vitro GI digestion were fermented in vitro by human gut microbiota, which was obtained from three healthy donors (22-28 years) without antibiotic treatment for 3 months before experimentation, and without a history of gastrointestinal diseases; a two-day polyphenol free diet was conducted before sample collection. All healthy donors provided written informed consent. Samples were preserved in an anaerobic tank immediately after collection, and then 
diluted with 10\% (v/v) PBS buffer and blended to acquire a suspension embodying $10 \%(w / v)$ phosphate buffer. The suspensions were used as the fermentation initiator.

\subsubsection{Fermentation Conditions}

Fermentation broth $(10 \mathrm{~mL})$ consisted of $45 \%$ fecal suspensions, $45 \%$ growth medium, and $10 \%$ legume residues collected from the in vitro GI digestion. Fermentation was started by mixing suspensions, fecal suspensions, and legume samples and incubated at $37^{\circ} \mathrm{C}$ at 200 strokes $/ \mathrm{min}$ for $48 \mathrm{~h}$. The samples were collected at $0,1,3,6,12,24,36$, and $48 \mathrm{~h}$, centrifuged, and $1 \mathrm{ml}$ was filtered through a $0.22 \mu \mathrm{m}$ PTFE membrane for HPLC analyses. The $\mathrm{pH}$ value of the fermentation broth was detected at each period. The samples were saved at $-80^{\circ} \mathrm{C}$ in a refrigerator until analysis.

\subsection{Determination of Total Phenolic Content (TPC) and Total Flavonoid Content (TFC)}

\subsubsection{Determination of TPC}

A Folin-Ciocalteu assay was used to determine the TPC, as described in previous methods [17]. In short, $25 \mu \mathrm{L}$ samples or gallic acid standards $(5,25,50,100,200,400,600 \mu \mathrm{g} / \mathrm{mL})$ were blended with Folin-Ciocalteu reagent $(125 \mu \mathrm{L}, 0.2 \mathrm{M})$ in a 96 well microplate and incubated in an incubator at $37^{\circ} \mathrm{C}$ for $6 \mathrm{~min} ; 125 \mu \mathrm{L} \mathrm{Na} \mathrm{CO}_{3}(10 \mathrm{~g} / 100 \mathrm{~mL})$ solution was added and reacted for $30 \mathrm{~min}$. An ELX800 microplate reader (BioTek Instruments, Inc., Winooski, VT, USA) was used to read the absorbance at $765 \mathrm{~nm}$. The TPC was calculated as milligrams of gallic acid equivalent (GAE, mg GAE/g DW).

\subsubsection{Determination of TFC}

An Erinitrit aluminum trichloride assay was used to determine the TFC [17]. In brief, $25 \mu \mathrm{L}$ of catechin standard $(5,25,50,100,200 \mu \mathrm{g} / \mathrm{mL})$ or bean extract was mixed with $110 \mu \mathrm{L} \mathrm{NaNO} 2(0.066 \mathrm{M})$ in a 96-well microplate and kept for $5 \mathrm{~min}$ at room temperature; $15 \mu \mathrm{L} \mathrm{AlCl}_{3}(0.75 \mathrm{M})$ was added and reacted at room temperature for $6 \mathrm{~min}$; then $100 \mu \mathrm{L} \mathrm{NaOH}(0.5 \mathrm{M})$ solution was added and reacted at $37^{\circ} \mathrm{C}$ for another $10 \mathrm{~min}$. An ELX800 microplate reader (BioTek Instruments, Inc., Winooski, VT, USA) was used to read the absorbance of the mixture at $510 \mathrm{~nm}$. The TFC was calculated as milligram catechin equivalent (CAE, mg CAE/g DW).

\subsection{Antioxidant Activity}

\subsubsection{ABTS Radical Scavenging Activity}

ABTS was assessed based on a previously reported method, with slight modifications [18]. ABTS working solution was prepared through blending $0.2 \mathrm{~mL} \mathrm{ABTS}^{+}$stock solution $(7.4 \mathrm{mM})$ with $0.2 \mathrm{~mL} \mathrm{~K}_{2} \mathrm{~S}_{2} \mathrm{O}_{8}(2.6 \mathrm{mM})$ in a ratio of $1: 1(v / v)$, and reacting for $12 \mathrm{~h}$ in the dark. The ABTS working solution was diluted with $80 \%$ ethanol to obtain the absorbance of $0.70 \pm 0.05$ at $734 \mathrm{~nm}$. Then, $200 \mu \mathrm{L}$ of ABTS working solution was mixed with $10 \mu \mathrm{L}$ of samples or Trolox standards $(20,40,50,60,70$, $80 \mu \mathrm{g} / \mathrm{mL}$ ) and incubated in a 96 well plate for $6 \mathrm{~min}$ in the dark. A Thermo Varioskan Flash Microplate Reader (Thermo Scientific, Waltham, MA, USA) was used to read the absorbance at $734 \mathrm{~nm}$. All samples were measured three times. The antioxidant activity was denoted as micromole Trolox equivalents (TE) per gram of dry weight (DW) (mmol TE/g DW).

\subsubsection{Ferric Reducing Antioxidant Power (FRAP Assay)}

For FRAP assay, the procedure was based on the method of Zhang B et al. [17]. Acetate buffer (300 mM), $\mathrm{FeCl}_{3} \cdot 6 \mathrm{H}_{2} \mathrm{O}(20 \mathrm{mM})$, and $10 \mathrm{mM} \mathrm{TPTZ}$ in $40 \mathrm{mM} \mathrm{HCl}$ were mixed at a ratio of 10:1:1 (v/v/v) and warmed to $37^{\circ} \mathrm{C}$ to prepare the fresh working solution. Then, $5 \mu \mathrm{L}$ of samples or $\mathrm{FeSO}_{4}$ standards $(0.03125,0.0625,0.125,0.25,0.5,1 \mathrm{mmol} / \mathrm{L})$ was reacted with $180 \mu \mathrm{L}$ working solution in a 96 well plate for $10 \mathrm{~min}$ at $37^{\circ} \mathrm{C}$. A Thermo Varioskan Flash Microplate (Thermo Scientific, Waltham, MA, USA) was 
used to measure the absorbance at $593 \mathrm{~nm}$. The antioxidant activity was denoted as $\mathrm{FeSO}_{4}$ equivalents (FE, mmol FE/g DW).

\subsection{Qualitative Analysis by UPLC-ESI-QTOF-MS/MS}

\subsubsection{Liquid Cromatographic Conditions}

Each of the samples was detected by a 1290 infinity series Ultra Performance Liquid Chromatography (UPLC) system (Agilent Technologies, Santa Clara, CA, USA) equipped with a diode array detector (DAD) and Agilent Eclipse Plus C18 column $(2.1 \mathrm{~mm} \times 100 \mathrm{~mm}, 1.8 \mu \mathrm{m})$, with a column heater set to $35^{\circ} \mathrm{C}$. The mobile phase was composed of $0.1 \%$ formic acid in de-ionized water (solvent A) and methanol (solvent $\mathrm{B}$ ), and passed at a flow rate of $0.3 \mathrm{~mL} / \mathrm{min}$ The linear gradient was as follows: $0-10 \mathrm{~min}, 5-10 \% \mathrm{~B} ; 10-30 \mathrm{~min}, 10-30 \% \mathrm{~B} ; 30-38 \mathrm{~min}, 30-50 \% \mathrm{~B} ; 38-43 \mathrm{~min}, 50 \% \mathrm{~B}$; 43-45 $\mathrm{min}, 50-5 \% \mathrm{~B}$. The UV-visible absorbance of the peak was monitored between 200 and $400 \mathrm{~nm}$. The injection volume was $5 \mu \mathrm{L}$.

\subsubsection{Mass Spectrometric Conditions}

An orthogonal acceleration quadrupole time-of-flight mass spectrometer (Agilent Technologies, Santa Clara, CA, USA) equipped with an Electron Spray Ionization (ESI) source was used for qualitative analysis. Phenolic compounds were monitored by the negative ion mode in a $\mathrm{m} / \mathrm{z}$ range from 50 to 1000. The mass capillary voltage was kept at $4.5 \mathrm{kV}$ and the flow rate of drying gas $\left(\mathrm{N}_{2}\right)$ was $10 \mathrm{~L} / \mathrm{min}$, with the temperature at $300{ }^{\circ} \mathrm{C}$. Nebulizer pressure, fragmentor voltage, and collision energy were set at $30 \mathrm{psi}, 175 \mathrm{~V}$, and $10-40 \mathrm{eV}$, respectively.

\subsection{Quantitative Analysis by HPLC-ESI-QqQ-MS}

\subsubsection{LC-MS Conditions}

The samples were quantified by HPLC mass spectrometry (MS) analysis. The sample was injected into an Agilent Eclipse Plus C18 column $(2.1 \mathrm{~mm} \times 100 \mathrm{~mm}, 1.8 \mu \mathrm{m})$. Then, $0.1 \%$ formic acid in de-ionized water (solvent $\mathrm{A}$ ) and methanol (solvent $\mathrm{B}$ ) were used as mobile phase. The elution gradient was as follows: $0 \mathrm{~min}, 5 \% \mathrm{~B} ; 18 \mathrm{~min}, 50 \% \mathrm{~B} ; 25 \mathrm{~min}, 95 \% \mathrm{~B} ; 28 \mathrm{~min}, 5 \%$ B. Other HPLC conditions were the same as for UPLC.

A triple quadrupole mass spectrometer (Agilent Technologies, Santa Clara, CA, USA) equipped with an ESI source was used for quantitative analysis. The instrument was utilized in the negative mode, and in multiple reactions monitoring (MRM) mode. The mass capillary voltage was kept at $+4.0 \mathrm{kV}$ and the drying gas flow rate was $11 \mathrm{~L} / \mathrm{min}$ with the temperature at $300^{\circ} \mathrm{C}$. Nebulizer pressure was set at $15 \mathrm{psi}$, and the fragment voltage and collision energy of each material was optimized.

\subsubsection{Calibration and Quantification of Phenolics}

A calibration curve was used to evaluate the linearity for 21 phenolic standards, which were subjected to accurate weighing $(1 \mathrm{mg})$, and dissolved in methanol $(1 \mathrm{~mL})$, then serially diluted to 10 , $25,50,100,150$, and $200 \mu \mathrm{g} / \mathrm{mL}$.

\subsection{Statistical Analysis}

All experiments were performed in triplicate, and the data were presented as mean value \pm standard deviations (SD). The limit of detection (LOD) and limit of quantitation (LOQ) were determined from the signal to noise ratio $(\mathrm{S} / \mathrm{R})$, considering the LOD and LOQ to be the lowest concentration capable of generating an $S / R \geq 3$ and $S / R \geq 10$, respectively. The data were analyzed by one-way analysis of variance (ANOVA), and Duncan's multiple range test was used to determine statistical differences on the level of significance at $P<0.05$. All statistical analyses were conducted using SPSS statistical software (Version 18.0, SPSS Inc., Armonk, New York, NY, USA). 


\section{Result and Discussion}

\subsection{Total Phenolics and Total Flavonoids of Legumes}

Total phenolic contents and total flavonoid contents of bound phenolics released by acid/alkaline hydrolysis in the different legumes are presented in (Table 1). Results show that the TPC value of the three legumes, obtained by acid hydrolysis treatment, was between 0.012 and $0.31 \mathrm{mg}$ GAE/g DW, while the TPCs obtained by the alkali hydrolysis treatment of the three legumes were remarkably higher than those by acid hydrolysis (1.79-2.27 mg GAE/g DW), indicating that alkaline hydrolysis was more efficient in releasing the bound phenolic compounds than acid hydrolysis, which is in accordance with earlier studies [19]. Moreover, the TPC of kidney bean $(2.07 \pm 0.09 \mathrm{mg} \mathrm{GAE} / \mathrm{g} \mathrm{DW})$, released through alkaline hydrolysis, was slightly lower than that of soybean $(2.27 \pm 0.30 \mathrm{mg} \mathrm{GAE} / \mathrm{g}$ DW), but without statistically significant difference. Interestingly, the soybean exhibited the lowest TPC of acid hydrolysis bound phenolics, only $0.012 \pm 0.001 \mathrm{mg}$ GAE/g DW. In addition, compared with the free phenolics (Table S1), there was no significant difference in TPC between bound phenolics (1.79-2.27 mg GAE/g DW) and free phenolics (2.04-2.48 mg GAE/g DW), which means that the main component of phenols in the three legumes were not only free phenolics, but that bound phenolics also occupied a large part. Wang et al. [20] also reported the contents of bound phenolics in 14 legumes, including soybeans, broad beans, and kidney beans, and ranging from $32.6 \%$ to $68.3 \%$.

Table 1. Total phenolic contents, total flavonoids content, and antioxidant activities of bound phenolics in legumes. ${ }^{\mathrm{A}}$.

\begin{tabular}{ccccc}
\hline Antioxidative Assay & Hydrolysis Method & Soybean & Vicia Faba & Kidney Bean \\
\hline TPC & acid & $0.012 \pm 0.001^{\mathrm{c}}$ & $0.18 \pm 0.01^{\mathrm{b}}$ & $0.31 \pm 0.01^{\mathrm{a}}$ \\
$(\mathrm{mg} \mathrm{GAE} / \mathrm{g}$ DW $)$ & alkaline & $2.27 \pm 0.30^{\mathrm{a}}$ & $1.79 \pm 0.12^{\mathrm{b}}$ & $2.07 \pm 0.09^{\mathrm{ab}}$ \\
TFC & acid & $0.006 \pm 0.001^{\mathrm{c}}$ & $0.09 \pm 0.03^{\mathrm{b}}$ & $0.17 \pm 0.02^{\mathrm{a}}$ \\
$(\mathrm{mg} \mathrm{CAE} / \mathrm{g}$ DW $)$ & alkaline & $0.13 \pm 0.03^{\mathrm{c}}$ & $0.35 \pm 0.05^{\mathrm{b}}$ & $0.76 \pm 0.03^{\mathrm{a}}$ \\
ABTS & acid & $1.54 \pm 0.04^{\mathrm{a}}$ & $1.11 \pm 0.06^{\mathrm{b}}$ & $1.52 \pm 0.04^{\mathrm{a}}$ \\
$(\mathrm{mg} \mathrm{TE} / \mathrm{g}$ DW $)$ & alkaline & $1.11 \pm 0.09^{\mathrm{c}}$ & $1.79 \pm 0.36^{\mathrm{b}}$ & $3.13 \pm 0.11^{\mathrm{a}}$ \\
FRAP & acid & $5.86 \pm 0.31^{\mathrm{c}}$ & $7.58 \pm 0.07^{\mathrm{b}}$ & $11.17 \pm 0.04^{\mathrm{a}}$ \\
$(\mathrm{mmol} \mathrm{FE} / \mathrm{g}$ DW) & alkaline & $4.57 \pm 0.25^{\mathrm{c}}$ & $15.24 \pm 1.10^{\mathrm{b}}$ & $30.77 \pm 1.77^{\mathrm{a}}$ \\
\hline
\end{tabular}

TPC, Total Phenolic Content; TFC, Total Flavonoid Content; ABTS, 2,2' -azinobis-(3-ethylbenzthiazoline-6-sulphonate; FRAP, Ferric Reducing Antioxidant Power. ${ }^{A}$ Results are expressed as mean \pm standard deviation of three replicates $(n=3)$; Values followed by the different letters $(\mathrm{a}, \mathrm{b}, \mathrm{c})$ within the same line are significantly different $(P<0.05)$.

Kim et al. [15] reported that the release of phenolic compounds under alkaline hydrolysis is better than that under acid hydrolysis. This may be due to alkaline hydrolysis being able to cleave the ester bonds between phenolic acid and polysaccharide, and reduce the loss of phenolic acids [19,21]. Among the three legumes, kidney beans have attracted more and more attention because of their excellent source of dietary antioxidants. Kan et al. [22] reported the TPC values of 26 kidney bean seed coats were between 0.25 and $35.11 \mathrm{mg}$ GAE/g DW. In this study, kidney bean contained dramatically higher TPC and TFC than the other two legumes, indicating that kidney bean is also an excellent source of bound phenolic.

\subsection{Identification and Quantification of Bound Phenolic Compounds of Three Legumes in Acid/Alkaline Hydrolysis}

The base peak chromatograms (BPC) obtained from the three legumes extracts are presented in (Figure 1). There were 9, 8, and 13 phenolic compounds released by acid hydrolysis in soybean, vicia faba, and kidney bean, while the phenolic compounds released with alkali hydrolysis were 12 , 18 , and 15 in soybean, vicia faba, and kidney bean, being 25\%,55\%, and 13\% superior, respectively. This result further confirmed that alkali hydrolysis was more effective than acid hydrolysis in releasing phenolic compounds from the three legumes.

The retention times, $\mathrm{MS}^{2}$ fragmentation pattern in negative, and UV spectra are shown in (Table 2). In summary, 36 bound phenolic were identified by comparison with the relevant standards, 
literature reports, or databases. The identified compounds can be classified into three groups, including phenolic acids and their derivatives, flavonoids and their derivatives, and other compounds. As seen in Table 2, 17 phenolic compounds, 9 flavonol compounds, and 10 other compounds were identified in the three legumes, among which 10 phenolic compounds were already identified in the soluble phenolic compounds (Table S2), including protocatechuic acid, p-coumaric acid, dihydroxybenzoic acid, catechin, ferulic acid, sinapic acid, daidzin, genistin, vitexin, and quercetin.

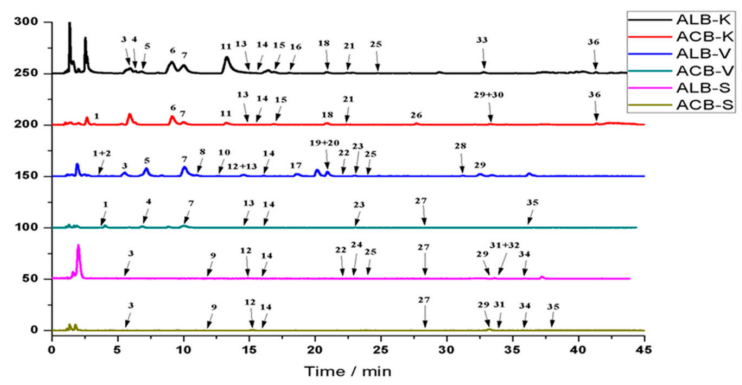

Figure 1. Base peak chromatogram (BPC) of bound phenolics in legumes. ACB-S: acid hydrolysis-bound phenolics of soybean; ALB-S: alkaline hydrolysis-bound phenolics of soybean; ACB-V: acid hydrolysis-bound phenolics of vicia faba; ALB-V: alkaline hydrolysis-bound phenolics of vicia faba; ACB-K: acid hydrolysisbound phenolics of kidney bean; ALB-K: alkaline hydrolysis-bound phenolics of kidney bean.

Table 2. Characterization of the bound phenolic constituents of legumes by UPLC-ESI-QTOF-MS2.

\begin{tabular}{|c|c|c|c|c|c|c|c|}
\hline $\begin{array}{l}\text { Peak } \\
\text { No. }\end{array}$ & $\frac{t_{R}}{(\min )}$ & $\begin{array}{l}\lambda_{\max } \\
(\mathrm{nm})\end{array}$ & Formula & {$[\mathbf{M}-\mathbf{H}]^{-}(m / z)$} & Major Fragment Ions $(\mathrm{m} / \mathrm{z}$ ) & Identification & Source \\
\hline \multicolumn{8}{|c|}{ Phenolic acids and derivatives } \\
\hline 1 & 3.555 & 206,273 & $\mathrm{C}_{7} \mathrm{H}_{6} \mathrm{O}_{5}$ & 169.0148 & $125.0205\left[\mathrm{M}-\mathrm{H}-\mathrm{CO}_{2}\right]^{-}$ & Gallic acid $^{a b c}$ & $\mathrm{~V} 1, \mathrm{~V} 2, \mathrm{~K} 1$ \\
\hline 2 & 3.623 & 206,273 & $\mathrm{C}_{9} \mathrm{H}_{10} \mathrm{O}_{5}$ & 197.006 & $153.0227\left[\mathrm{M}-\mathrm{H}-\mathrm{CO}_{2}\right]^{-}$ & Syringic acid $^{a b}$ & V2 \\
\hline 3 & 5.503 & 206,273 & $\mathrm{C}_{7} \mathrm{H}_{6} \mathrm{O}_{4}$ & 153.0189 & $\begin{array}{c}109.0261\left[\mathrm{M}-\mathrm{H}-\mathrm{CO}_{2}\right]^{-} \\
125.0214[\mathrm{M}-\mathrm{H}-\mathrm{CO}]^{-}\end{array}$ & Procatechuic acid ${ }^{a b c}$ & $\begin{array}{l}\mathrm{S} 1, \mathrm{~S} 2, \mathrm{~V} 2, \\
\mathrm{~K} 1, \mathrm{~K} 2\end{array}$ \\
\hline 4 & 6.86 & 261,292 & $\mathrm{C}_{7} \mathrm{H}_{6} \mathrm{O}_{4}$ & 153.0191 & $109.0286\left[\mathrm{M}-\mathrm{H}-\mathrm{CO}_{2}\right]^{-}$ & Dihydroxybenzoic acid ${ }^{a b}$ & $\mathrm{~V} 1, \mathrm{~K} 2$ \\
\hline 5 & 7.168 & 291,208 & $\mathrm{C}_{9} \mathrm{H}_{10} \mathrm{O}_{4}$ & 181.012 & $\begin{array}{c}153.0190[\mathrm{M}-\mathrm{H}-\mathrm{CO}]^{-} \\
135.0087\left[\mathrm{M}-\mathrm{H}-\mathrm{CO}-\mathrm{H}_{2} \mathrm{O}\right]^{-} \\
162.9992\left[\mathrm{M}-\mathrm{H}-\mathrm{H}_{2} \mathrm{O}\right]^{-}\end{array}$ & Hydroxyphenyllactic acid ${ }^{b}$ & V2, K2 \\
\hline 6 & 9.11 & 280,311 & $\mathrm{C}_{7} \mathrm{H}_{6} \mathrm{O}_{3}$ & 137.0222 & 109.0461[M-H-CO] ${ }^{-}$ & Hydroxybenzoic acid $^{\text {a }}$ & $\mathrm{K} 1, \mathrm{~K} 2$ \\
\hline 7 & 9.996 & 280,310 & $\mathrm{C}_{7} \mathrm{H}_{6} \mathrm{O}_{3}$ & 137.0225 & $\begin{array}{c}119.0129\left[\mathrm{M}-\mathrm{H}-\mathrm{H}_{2} \mathrm{O}\right]^{-} \\
108.0238[\mathrm{M}-\mathrm{H}-\mathrm{CHO}]^{-}\end{array}$ & Hydroxybenzoic acid ${ }^{a}$ & $\begin{array}{l}\text { V1, V2, K2, } \\
\text { K2 }\end{array}$ \\
\hline 9 & 11.181 & 256 & $\mathrm{C}_{7} \mathrm{H}_{6} \mathrm{O}_{3}$ & 137.0254 & $\begin{array}{c}119.0115\left[\mathrm{M}-\mathrm{H}-\mathrm{H}_{2} \mathrm{O}\right]^{-} \\
108.0205[\mathrm{M}-\mathrm{H}-\mathrm{CHO}]^{-}\end{array}$ & Hydroxybenzoic acid ${ }^{a}$ & S1, S2 \\
\hline 14 & 15.614 & 270 & $\mathrm{C}_{10} \mathrm{H}_{10} \mathrm{O}_{4}$ & 193.0144 & $133.2188\left[\mathrm{M}-\mathrm{H}-\mathrm{C}_{2} \mathrm{H}_{4} \mathrm{O}_{2}\right]^{-}$ & Ferulic acid abc & $\mathrm{V} 2, \mathrm{~K} 2$ \\
\hline 16 & 18.01 & 268 & $\mathrm{C}_{11} \mathrm{H}_{12} \mathrm{O}_{5}$ & 223.021 & & Sinapic acid ${ }^{b c}$ & K2 \\
\hline 18 & 20.836 & 264,294 & $\mathrm{C}_{8} \mathrm{H}_{8} \mathrm{O}_{4}$ & 167.0326 & $148.8648\left[\mathrm{M}-\mathrm{H}-\mathrm{H}_{2} \mathrm{O}\right]^{-}$ & 4-Hydroxyphenylglycolic acid ${ }^{\text {a }}$ & $\mathrm{K} 1, \mathrm{~K} 2$ \\
\hline 19 & 21.018 & 273 & $\mathrm{C}_{11} \mathrm{H}_{12} \mathrm{O}_{5}$ & 223.0358 & 179.0437[M-H-CO $]^{-}$ & Sinapic acid $^{\text {ac }}$ & $\mathrm{V} 2$ \\
\hline 22 & 22.89 & 296 & $\mathrm{C}_{9} \mathrm{H}_{8} \mathrm{O}_{3}$ & 163.0358 & $119.0500\left[\mathrm{M}-\mathrm{H}-\mathrm{CO}_{2}\right]^{-}$ & Coumaric acid ${ }^{a b}$ & $\mathrm{~S} 2, \mathrm{~V} 2$ \\
\hline 24 & 23.762 & 290 & $\mathrm{C}_{16} \mathrm{H}_{18} \mathrm{O}_{9}$ & 353.1136 & $190.8992\left[\mathrm{M}-\mathrm{H}-\mathrm{C}_{6} \mathrm{H}_{10} \mathrm{O}_{5}\right]^{-}$ & Chlorogenic acid ${ }^{a b c}$ & S2 \\
\hline 25 & 24.728 & 290 & $\mathrm{C}_{9} \mathrm{H}_{8} \mathrm{O}_{3}$ & 163.0402 & $119.0494\left[\mathrm{M}-\mathrm{H}-\mathrm{CO}_{2}\right]^{-}$ & p-Coumaric acid abc & $\mathrm{S} 2, \mathrm{~V} 2, \mathrm{~K} 2$ \\
\hline 29 & 33.099 & 271 & & 343.2033 & $297.1303,163.0575$ & Coumaric acid derivative $^{a}$ & $\begin{array}{l}\text { S1, S2, } \\
\mathrm{V} 2, \mathrm{~K} 1\end{array}$ \\
\hline 35 & 36.65 & 268 & $\mathrm{C}_{9} \mathrm{H}_{8} \mathrm{O}_{3}$ & 162.8382 & $119.0492\left[\mathrm{M}-\mathrm{H}-\mathrm{CO}_{2}\right]^{-}$ & Coumaric acid $^{a b}$ & $\mathrm{~S} 1, \mathrm{~V} 1$ \\
\hline 27 & 28.416 & 282 & $\mathrm{C}_{21} \mathrm{H}_{20} \mathrm{O}_{9}$ & 415.1493 & $\begin{array}{c}\text { Flavonoids } \\
\text { Isoflavones and derivatives } \\
253.0198\left[\mathrm{M}-\mathrm{C}_{6} \mathrm{H}_{10} \mathrm{O}_{5}\right]^{-}\end{array}$ & Daidzin $^{\text {abc }}$ & $\mathrm{S} 1, \mathrm{~S} 2$ \\
\hline 31 & 33.69 & 274 & $\mathrm{C}_{21} \mathrm{H}_{20} \mathrm{O}_{10}$ & 431.0939 & $269.0404\left[\mathrm{M}-\mathrm{H}-\mathrm{C}_{6} \mathrm{H}_{10} \mathrm{O}_{5}\right]^{-}$ & Genistin $^{\text {ac }}$ & $\mathrm{S} 1, \mathrm{~S} 2$ \\
\hline & & & & & Flavones and derivatives & & \\
\hline 20 & 20.956 & 280 & $\mathrm{C}_{15} \mathrm{H}_{10} \mathrm{O}_{5}$ & 269.064 & & Trihydroxyflavone ${ }^{a}$ & V2 \\
\hline 32 & 34.043 & 298 & $\mathrm{C}_{21} \mathrm{H}_{20} \mathrm{O}_{10}$ & 431.093 & $477.1000[\mathrm{M}-\mathrm{H}+\mathrm{HCOOH}]^{-}$ & Vitexin $^{a b c}$ & $\mathrm{~S} 2$ \\
\hline
\end{tabular}


Table 2. Cont.

\begin{tabular}{|c|c|c|c|c|c|c|c|}
\hline Peak & $t_{R}$ & $\lambda_{\max }$ & \multirow{2}{*}{ Formula } & \multirow{2}{*}[\mathrm{M}-\mathrm{H}]{$^{-}(m / z)$} & \multirow[t]{2}{*}{ Major Fragment Ions $(\mathrm{m} / \mathrm{z})$} & \multirow{2}{*}{ Identification } & \multirow{2}{*}{ Source } \\
\hline No. & $(\min )$ & $(\mathrm{nm})$ & & & & & \\
\hline 36 & 41.448 & 265 & $\mathrm{C}_{15} \mathrm{H}_{10} \mathrm{O}_{7}$ & 301.0318 & & Quercetin abc & $\mathrm{K} 1, \mathrm{~K} 2$ \\
\hline \multicolumn{8}{|c|}{ Flavonols and derivatives } \\
\hline 21 & 22.425 & 294 & $\mathrm{C}_{15} \mathrm{H}_{10} \mathrm{O}_{8}$ & 317.0303 & $\begin{array}{l}\left.\text { 190.9986[M-H-C }{ }_{6} \mathrm{H}_{6} \mathrm{O}_{3}\right]^{-} \\
163.0008\left[\mathrm{M}-\mathrm{H}-\mathrm{C}_{7} \mathrm{H}_{6} \mathrm{O}_{4}\right]^{-}\end{array}$ & Myricetin ab & $\mathrm{K} 1, \mathrm{~K} 2$ \\
\hline \multicolumn{8}{|c|}{ Flavanones and derivatives } \\
\hline 26 & 27.935 & 267 & $\mathrm{C}_{15} \mathrm{H}_{12} \mathrm{O}_{8}$ & 319.04 & 183.027 & Ampelopsin ${ }^{\mathrm{a}}$ & K1 \\
\hline 30 & 33.285 & 268 & $\mathrm{C}_{16} \mathrm{H}_{14} \mathrm{O}_{8}$ & 333.1053 & & Hovenitin $\mathrm{I}^{\mathrm{a}}$ & K1 \\
\hline \multicolumn{8}{|c|}{ Flavanes and derivatives } \\
\hline 13 & 15.484 & 278 & $\mathrm{C}_{15} \mathrm{H}_{14} \mathrm{O}_{6}$ & 289.0666 & 109.1011[M-H- $\left.\mathrm{C}_{9} \mathrm{H}_{12} \mathrm{O}_{4}\right]^{-}$ & Catechin abc & $\begin{array}{l}\text { V2, V1, } \\
\text { K1, K2 }\end{array}$ \\
\hline \multicolumn{8}{|c|}{ Other compounds } \\
\hline 8 & 11.134 & 208,273 & $\mathrm{C}_{8} \mathrm{H}_{10} \mathrm{O}_{3}$ & 153.0183 & $\begin{array}{c}123.0441\left[\mathrm{M}-\mathrm{H}-\mathrm{CH}_{2} \mathrm{O}\right]^{-}, \\
125.6422[\mathrm{M}-\mathrm{H}-\mathrm{CO}]^{-}\end{array}$ & Hydroxytyrosol ${ }^{b}$ & V2 \\
\hline 10 & 12.667 & 285 & $\mathrm{C}_{9} \mathrm{H}_{6} \mathrm{O}_{3}$ & 161.0776 & $117.0551\left[\mathrm{M}-\mathrm{H}-\mathrm{CO}_{2}\right]^{-}$ & 4-Hydroxycoumarin ${ }^{b}$ & V2 \\
\hline 11 & 13.233 & 283 & $\mathrm{C}_{7} \mathrm{H}_{6} \mathrm{O}_{2}$ & 121.0277 & & m-Hydroxybenzaldehyde ${ }^{a b}$ & K1 \\
\hline 12 & 14.701 & 284 & $\mathrm{C}_{7} \mathrm{H}_{6} \mathrm{O}_{2}$ & 121.0295 & & p-Hydroxybenzaldehyde ab & $\mathrm{S} 1, \mathrm{~S} 2, \mathrm{~V} 2$ \\
\hline 15 & 16.83 & 287 & $\mathrm{C}_{8} \mathrm{H}_{10} \mathrm{O}_{3}$ & 153.0174 & $\begin{array}{c}125.0286[\mathrm{M}-\mathrm{H}-\mathrm{CO}]^{-} \\
123.0128\left[\mathrm{M}-\mathrm{H}-\mathrm{CH}_{2} \mathrm{O}\right]^{-}\end{array}$ & Hydroxytyrosol $^{\mathrm{b}}$ & $\mathrm{K} 1, \mathrm{~K} 2$ \\
\hline 17 & 18.357 & 287 & $\mathrm{C}_{8} \mathrm{H}_{10} \mathrm{O}_{3}$ & 153.0203 & $\begin{array}{c}125.0286[\mathrm{M}-\mathrm{H}-\mathrm{CO}]^{-} \\
123.0128\left[\mathrm{M}-\mathrm{H}-\mathrm{CH}_{2} \mathrm{O}\right]^{-}\end{array}$ & Hydroxytyrosol $^{\mathrm{b}}$ & V2 \\
\hline 23 & 23.01 & 283 & $\mathrm{C}_{14} \mathrm{H}_{8} \mathrm{O}_{4}$ & 239.0894 & $195.1382\left[\mathrm{M}-\mathrm{H}-\mathrm{CO}_{2}\right]^{-}$ & Alizarin $^{\text {a }}$ & $\mathrm{V} 1, \mathrm{~V} 2$ \\
\hline 28 & 31.256 & 282 & $\mathrm{C}_{13} \mathrm{H}_{14} \mathrm{O}_{3}$ & 217.1048 & $172.8927\left[\mathrm{M}-\mathrm{H}-\mathrm{CO}_{2}\right]^{-}$ & EUPATORIOCHROMENE $^{\mathrm{b}}$ & V2 \\
\hline 33 & 36.732 & 264 & $\mathrm{C}_{18} \mathrm{H}_{24} \mathrm{O}_{3}$ & 287.15 & $\begin{array}{c}269.1377\left[\mathrm{M}-\mathrm{H}_{-} \mathrm{H}_{2} \mathrm{O}\right]^{-} \\
227.1307,209.5160\end{array}$ & 2-Hydroxyestradiol ${ }^{\mathrm{b}}$ & K2 \\
\hline 34 & 35.801 & 295 & $\mathrm{C}_{13} \mathrm{H}_{10} \mathrm{O}_{6}$ & 261.1502 & $\begin{array}{c}125.0975\left[\mathrm{M}-\mathrm{H}-\mathrm{C}_{7} \mathrm{H}_{4} \mathrm{O}_{3}\right]^{-} \\
187.0988\end{array}$ & Maclurin $^{a}$ & S1, S2 \\
\hline
\end{tabular}

S1: acid hydrolysis-bound phenolics of soybean; S2: alkaline hydrolysis-bound phenolics of soybean; V1: acid hydrolysisbound phenolics of vicia faba; V2: alkaline hydrolysis-bound phenolics of vicia faba; K1: acid hydrolysis-bound phenolics of kidney bean; K2: alkaline hydrolysis-bound phenolics of kidney bean. ${ }^{a}$ Compared with the literature. ${ }^{b}$ Compared with MSn data, data bases, and/or characteristic UV spectra. ${ }^{c}$ Compared with an authentic standard.

\subsubsection{Phenolic Acids}

A total of 17 phenolic acids and their derivatives were authenticated in the three legumes, most of which were observed in both kidney bean and vicia faba, but only compounds 3, 9, 24, 25, 29, and 35 were found in soybean. As shown in Table 2, compounds $1\left(t_{R} 3.555 \mathrm{~min}, \mathrm{~m} / z\right.$ 169), $3\left(t_{R} 5.503 \mathrm{~min}\right.$, $\mathrm{m} / \mathrm{z}$ 153), $14\left(\mathrm{t}_{\mathrm{R}} 15.614 \mathrm{~min}, \mathrm{~m} / \mathrm{z}\right.$ 193), $16\left(\mathrm{t}_{\mathrm{R}} 18.010 \mathrm{~min}, \mathrm{~m} / \mathrm{z} 223\right), 24\left(\mathrm{t}_{\mathrm{R}} 23.762 \mathrm{~min}, \mathrm{~m} / \mathrm{z} 353\right)$, and 25 $\left(t_{R} 24.728 \mathrm{~min}, \mathrm{~m} / \mathrm{z} 163\right.$ ) were authenticated as gallic acid, protocatechuic acid, ferulic acid, sinapic acid, chlorogenic acid, and p-coumaric acid, respectively, through comparison with the retention time and mass spectrum data of the respective standard compounds. Compound $19\left(\mathrm{t}_{\mathrm{R}} 21.018 \mathrm{~min}, \mathrm{~m} / \mathrm{z} 223\right)$ and compound $16\left(t_{R} 18.010 \mathrm{~min}, \mathrm{~m} / \mathrm{z} 223\right)$ were tentatively authenticated as the isomer of sinapic acid, due to the same molecular ion but a different retention time. Compound $2\left(t_{R} 3.623 \mathrm{~min}, \mathrm{~m} / z\right.$ 197), with a fragment ion at $m / z 153\left[\mathrm{M}-\mathrm{H}-\mathrm{CO}^{2}\right]^{-}$, corresponded to syringic acid [23]. Compound $4\left(\mathrm{t}_{\mathrm{R}} 6.860 \mathrm{~min}\right.$, $\mathrm{m} / \mathrm{z}$ 153), exhibiting fragment ions at $\mathrm{m} / \mathrm{z} 109\left[\mathrm{M}-\mathrm{H}-\mathrm{CO}^{2}\right]^{-}$, was characterized as dihydroxybenzoic acid [24]. Compound 5 ( $\mathrm{t}_{\mathrm{R}} 7.168 \mathrm{~min}, \mathrm{~m} / \mathrm{z}$ 181) was regarded as hydroxyphenyllactic acid, due to its fragment ions at $m / z 153.0190[\mathrm{M}-\mathrm{H}-\mathrm{CO}]^{-}, 135\left[\mathrm{M}-\mathrm{H}-\mathrm{CO}-\mathrm{H}_{2} \mathrm{O}\right]^{-}$, and $162\left[\mathrm{M}-\mathrm{H}-\mathrm{H}_{2} \mathrm{O}\right]^{-}$, and by referring to the Metlin online database. Based on a reported study [14], compounds 6, 7, and $9\left(t_{R} 9.110,9.996\right.$ and $11.181 \mathrm{~min}$ ), which displayed the same $[\mathrm{M}-\mathrm{H}]^{-}$ion at $\mathrm{m} / \mathrm{z} 137$, were preliminarily characterized as hydroxybenzoic acid. Compound $18\left(t_{R} 20.836 \mathrm{~min}, \mathrm{~m} / \mathrm{z} 167\right)$ revealed a major fragment ion at $\mathrm{m} / \mathrm{z}$ $148.8648\left[\mathrm{M}-\mathrm{H}-\mathrm{H}_{2} \mathrm{O}\right]^{-}$, which was authenticated as 4-hydroxyphenylglycolic acid [25]. Compounds 22 and $35\left(t_{R} 22.890\right.$ and $\left.36.650 \mathrm{~min}, \mathrm{~m} / \mathrm{z} 163\right)$ were initially regarded as coumaric acid based on a literature comparison [26]. Compound $29\left(t_{R} 33.099 \mathrm{~min}, \mathrm{~m} / z\right.$ 343) was authenticated as a coumaric acid derivative, classified according to its characteristic fragment ion at $m / z 163$. 


\subsubsection{Flavonoids}

A total of nine flavonoids, including isoflavones, flavones, flavonols, flavanones, flavanes, and their derivatives were observed in the three legumes, among which compounds 27 (daidzin), 31 (genistin), and 32 (vitexin) were only found in soybean, compound 36 (quercetin), 21 (myricetin), 26 (ampelopsin), and 30 (hovenitin I) were only present in kidney bean, compound 30 was only characterized in vicia faba, while compound 13 (catechin) was found in both kidney bean and vicia faba.

Compounds $13\left(\mathrm{t}_{\mathrm{R}} 15.484 \mathrm{~min}, \mathrm{~m} / \mathrm{z} 289\right), 27\left(\mathrm{t}_{\mathrm{R}} 28.416 \mathrm{~min}, \mathrm{~m} / \mathrm{z} 415\right), 31\left(\mathrm{t}_{\mathrm{R}} 33.690 \mathrm{~min}, \mathrm{~m} / \mathrm{z} 431\right)$, $32\left(t_{R} 34.043 \mathrm{~min}, \mathrm{~m} / \mathrm{z} 431\right)$ and $36\left(t_{R} 41.448 \mathrm{~min}, \mathrm{~m} / \mathrm{z} 301\right)$ were characterized as catechin, daidzin, genistin, vitexin, and quercetin, respectively, with an authentic standard. Compounds $20\left(t_{R} 20.956 \mathrm{~min}\right.$, $\mathrm{m} / \mathrm{z} 269), 21\left(\mathrm{t}_{\mathrm{R}} 22.425 \mathrm{~min}, \mathrm{~m} / \mathrm{z} 317\right), 26\left(\mathrm{t}_{\mathrm{R}} 27.935 \mathrm{~min}, \mathrm{~m} / \mathrm{z} 319\right)$, and $30\left(\mathrm{t}_{\mathrm{R}} 33.285 \mathrm{~min}, \mathrm{~m} / \mathrm{z} 333\right)$ were preliminarily authenticated as trihydroxyflavone, myricetin, ampelopsin, and hovenitin I, respectively, by comparison with other works $[14,23]$. Under this condition, some non-phenolic substances were also detected. After comparing with the Metlin online database, compounds 8, 15, and $17\left(\mathrm{t}_{\mathrm{R}} 11.134,16.830\right.$, $18.357 \mathrm{~min}, \mathrm{~m} / \mathrm{z} 153$ ), with the same $[\mathrm{M}-\mathrm{H}]^{-}$ion at $\mathrm{m} / \mathrm{z} 153$ and typical fragment ions at $123\left[\mathrm{M}-\mathrm{H}-\mathrm{CH}_{2} \mathrm{O}\right]^{-}$ and $125[\mathrm{M}-\mathrm{H}-\mathrm{CO}]^{-}$, were tentatively classified as hydroxytyrosol, whereas compound $10\left(\mathrm{t}_{\mathrm{R}} 12.667 \mathrm{~min}\right.$, $\mathrm{m} / \mathrm{z} 161)$, compound $28\left(\mathrm{t}_{\mathrm{R}} 31.256 \mathrm{~min}, \mathrm{~m} / \mathrm{z} 217\right)$, and compound $33\left(\mathrm{t}_{\mathrm{R}} 36.732 \mathrm{~min}, \mathrm{~m} / \mathrm{z} 287\right.$ ) were regarded as 4-hydroxycoumarin, eupatoriochromene, and 2-hydroxyestradiol, respectively. Compound 11 $\left(t_{R} 13.223 \mathrm{~min}\right.$ ) carried the same molecular ion at $\mathrm{m} / \mathrm{z} 121$, but its retention time was earlier than compound $12\left(t_{R} 14.701 \mathrm{~min}\right.$ ), and was identified as m-hydroxybenzaldehyde, and compound 12 as p-hydroxybenzaldehyde [27]. Compound $23\left(t_{R} 23.010 \mathrm{~min}, \mathrm{~m} / \mathrm{z} 239\right)$ displayed a fragment ion at $\mathrm{m} / \mathrm{z}$ 195, suggesting that it could be alizari [28]. Compound $34\left(t_{R} 35.801 \mathrm{~min}, \mathrm{~m} / \mathrm{z} 261\right)$ carried a fragment ion at $m / z 125$ and 187, and was characterized as maclurin, according to previously reported data [29].

The results showed that the kind of bound phenolic compounds found differed greatly in soybean, vicia faba, and kidney bean. More specifically, compounds 9, 24, 27, 31, 32, and 34 (Hydroxybenzoic acid, chlorogenic acid, daidzin, genistin, vitexin, and maclurin, respectively) were only detected in the hydrolysates of soybean, while compounds 2, 19, 20, 8, 10, 23, and 28 (syringic acid, sinapic acid, trihydroxyflavone, hydroxytyrosol, 4-hydroxycoumarin, alizarin, and eupatoriochromene, respectively) were only present in the hydrolysates of vicia faba, and compounds $6,16,18,36,21,26,30,11$, and 33 (Hydroxybenzoic acid, sinapic acid, 4-hydroxyphenylglycolic acid, quercetin, myricetin, ampelopsin, hovenitin I, and 2-hydroxyestradiol, respectively) were only found in the hydrolysates of kidney bean. The type of bound phenol compound released by hydrolysis was related to the method of hydrolysis, in addition to the kind of legume. There were differences in the composition of different methods of extracting bound phenolic compounds; some phenolic substances could only be detected from acid hydrolysates or alkali hydrolysates, such as compounds 2, 5, 14, 16, 19, 24, 25, 20, 32, 8,10 , and 33 (syringic acid, hydroxyphenyl lactic acid, ferulic acid, sinapic acid, chlorogenic acid, p-coumaric acid trihydroxyflavone, vitexin, hydroxytyrosol, 4-hydroxycoumarin, eupatoriochromene, and 2-hydroxyestradiol, respectively) were only found in alkaline hydrolysis, whereas compounds 26 , 30, and 11 (Ampelopsin, hovenitin I, and m-hydroxybenzaldehyde, respectively) were only present in acid hydrolysis. This might be due to alkaline hydrolysis being able to efficiently hydrolyze the ether bond or ester bond between phenolic compounds and food substrates, while acid hydrolysis prefers to hydrolyze glycosidic bonds $[30,31]$. Moreover, the phenolic extracts after alkaline hydrolysis and acid hydrolysis were almost all individual phenols, and with few glycoside phenols, while the soluble phenols have many types of glycoside phenols (Table S1).

\subsubsection{Quantitative Analysis of Bound Phenolic Compounds}

Triple quadrupole mass spectrometer and external standard methods were used to determine the content of bound phenolic compounds in the legumes. Due to the difficulty of purchasing the standard substances, only 20 chemical components, including 7 phenolic acids and 13 flavonoids were analyzed.

As shown in (Table 3), protocatechuic acid $(7.83-46.87 \mu \mathrm{g} / \mathrm{g} D W)$ was the major phenolic acid in the three legumes, followed by p-hydroxybenzoic acid $(0.20-20.74 \mu \mathrm{g} / \mathrm{g} \mathrm{DW})$, and p-coumaric 
acid (0.66-3.84 $\mu \mathrm{g} / \mathrm{g} \mathrm{DW})$. More specifically, the content of gallic acid was highest in kidney bean $(18.58 \mu \mathrm{g} / \mathrm{g}$ DW in acid hydrolysis and $9.57 \mu \mathrm{g} / \mathrm{g}$ DW in alkaline hydrolysis), but it was found in small amounts in the acid-hydrolyzed vicia faba $(0.32 \mu \mathrm{g} / \mathrm{g}$ DW $)$. Chlorogenic acid was only found in the alkaline hydrolyzed product of soybean $(0.84 \mu \mathrm{g} / \mathrm{g}$ DW). Moreover, the phenolic acids produced by acid hydrolysis were obviously higher than by alkaline hydrolysis in kidney bean, among which the protocatechuic acid, sinapic acid, and gallic acid obtained by acid hydrolysis $(16.13,0.2$ and $18.58 \mu \mathrm{g} / \mathrm{g}$ DW, respectively) were two times as much as that of alkaline hydrolysis $(7.83,0.08$ and $9.57 \mu \mathrm{g} / \mathrm{g}$ DW, respectively), while the content of p-coumaric acid in acid hydrolysis $(2.13 \mu \mathrm{g} / \mathrm{g} \mathrm{DW})$ was three times that of alkaline hydrolysis $(0.8 \mu \mathrm{g} / \mathrm{g} \mathrm{DW})$, and the p-hydroxybenzoic acid and ferulic acid released by acid hydrolysis ( 2.14 and $0.96 \mu \mathrm{g} / \mathrm{g}$ DW respectively) were as much as 10 times that of alkaline hydrolysis $(0.2$ and $0.19 \mu \mathrm{g} / \mathrm{g}$ DW respectively). This result indicated that the phenolic acids in kidney bean mentioned above may be released mainly by breaking glycosidic bonds and solubilizing sugar.

Table 3. Quantitative results of bound phenolics by HPLC-ESI-QqQ-MS a .

\begin{tabular}{|c|c|c|c|c|c|c|}
\hline \multirow{2}{*}{ Compounds } & \multicolumn{2}{|c|}{ Soybean $(\mu g / g$ DW) } & \multicolumn{2}{|c|}{ Vicia Faba ( $\mu$ g/g DW) } & \multicolumn{2}{|c|}{ Kidney Bean ( $\mu \mathrm{g} / \mathrm{g}$ DW) } \\
\hline & $\begin{array}{c}\text { Acid } \\
\text { Hydrolysis }\end{array}$ & $\begin{array}{l}\text { Alkaline } \\
\text { Hydrolysis }\end{array}$ & $\begin{array}{c}\text { Acid } \\
\text { Hydrolysis }\end{array}$ & $\begin{array}{l}\text { Alkaline } \\
\text { Hydrolysis }\end{array}$ & $\begin{array}{c}\text { Acid } \\
\text { Hydrolysis }\end{array}$ & $\begin{array}{l}\text { Alkaline } \\
\text { Hydrolysis }\end{array}$ \\
\hline \multicolumn{7}{|c|}{ Phenolic Acids } \\
\hline p-hydroxybenzoic acid & $2.11 \pm 0.02$ & $2.01 \pm 0.23$ & $19.96 \pm 0.42$ & $20.74 \pm 0.42$ & $2.14 \pm 0.14$ & $0.20 \pm 0.04$ \\
\hline procatechuic acid & $8.69 \pm 0.02$ & $9.87 \pm 0.21$ & $46.87 \pm 0.13$ & $31.58 \pm 0.36$ & $16.13 \pm 1.11$ & $7.83 \pm 0.11$ \\
\hline sinapic acid & $0.16 \pm 0.01$ & $0.03 \pm 0.01$ & $0.25 \pm 0.04$ & $0.26 \pm 0.01$ & $0.20 \pm 0.04$ & $0.08 \pm 0.03$ \\
\hline gallic acid & $\mathrm{Nd}$ & $\mathrm{Nd}$ & $0.32 \pm 0.01$ & $\mathrm{Nd}$ & $18.58 \pm 0.68$ & $9.57 \pm 0.10$ \\
\hline p-coumaric acid & $3.09 \pm 0.07$ & $3.84 \pm 0.15$ & $0.66 \pm 0.01$ & $0.74 \pm 0.01$ & $2.13 \pm 0.08$ & $0.80 \pm 0.02$ \\
\hline \multicolumn{7}{|c|}{ Flavonoids } \\
\hline \multicolumn{7}{|c|}{ Isoflavones } \\
\hline glycitin & $\mathrm{Nd}$ & $0.82 \pm 0.03$ & nd & nd & nd & nd \\
\hline \multicolumn{7}{|c|}{ Flavones } \\
\hline quercetin & $\mathrm{Nd}$ & $0.18 \pm 0.01$ & $4.31 \pm 0.07$ & $1.79 \pm 0.03$ & $0.20 \pm 0.02$ & $0.33 \pm 0.01$ \\
\hline hyperoside & $\mathrm{Nd}$ & $\mathrm{Nd}$ & nd & nd & nd & nd \\
\hline rutin & $\mathrm{Nd}$ & $0.28 \pm 0.14$ & nd & $0.20 \pm 0.01$ & nd & $1.30 \pm 0.02$ \\
\hline vitexin & 0.01 & $0.06 \pm 0.01$ & 0.02 & nd & $0.06 \pm 0.01$ & $0.05 \pm 0.01$ \\
\hline \multicolumn{7}{|c|}{ Flavanones } \\
\hline naringenin & $0.07 \pm 0.01$ & $0.02 \pm 0.01$ & 0.01 & nd & nd & 0.01 \\
\hline \multicolumn{7}{|c|}{ Flavanes } \\
\hline catechin & $\mathrm{Nd}$ & $\mathrm{Nd}$ & $7.97 \pm 0.40$ & $17.54 \pm 0.22$ & $0.84 \pm 0.02$ & $51.59 \pm 1.18$ \\
\hline Total & $22.68 \pm 0.24$ & $25.98 \pm 1.15$ & $82.06 \pm 0.82$ & $73.92 \pm 1.15$ & $41.24 \pm 2.22$ & $71.95 \pm 1.55$ \\
\hline
\end{tabular}

As for the flavonoid contents, isoflavones were mainly detected in soybeans; daidzin and genistin were the main isoflavones in soybean (ranging from 3.54 to $4.17 \mu \mathrm{g} / \mathrm{g} \mathrm{DW}$ ). For flavones, hyperoside was not measured in the three legumes, and quercetin showed a highest concentration at $4.13 \mu \mathrm{g} / \mathrm{g}$ DW in the acid hydrolysis of vicia faba. More specifically, rutin was only found in the alkaline hydrolysates of the three legumes. As for flavanes, catechin showed the highest concentration at $51.59 \mu \mathrm{g} / \mathrm{g}$ DW in the alkaline hydrolysis of kidney bean, followed by the alkaline hydrolysis of vicia faba ( $17.54 \mu \mathrm{g} / \mathrm{g} \mathrm{DW})$, however, it was not found in the soybean, indicating that rutin and catechin are mainly covalently bonded to the cell wall by ester bonds and ether bonds.

\subsection{The Antioxidant Activities of Legumes}

The antioxidant activities of bound phenolic compounds in the three legumes were studied by ABTS and FRAP in vitro (Table 1). As indicated, the ABTS value and FRAP value of insoluble-bound phenolic fractions in the three legumes ranged from 1.11 to $3.13 \mathrm{mg} \mathrm{TE} / \mathrm{g} \mathrm{DW}$, and 4.57 to $30.77 \mathrm{mmol}$ 
FE/g DW, respectively. Similar to the trend of TPC, the alkaline hydrolysis treatment showed remarkably higher ABTS and FRAP in vicia faba and kidney bean when compared with acid hydrolysis treatment, further verifying that alkaline hydrolysis is a better method of releasing bound antioxidants from these two legumes. However, soybeans showed the opposite trend to kidney beans and vicia faba, the acid hydrolysis treatment exhibited higher ABTS and FRAP $(1.54 \pm 0.04 \mathrm{mg}$ TE/g DW, $5.86 \pm 0.31 \mathrm{mg}$ TE/g $\mathrm{DW}$, respectively) in soybeans when compared to alkaline hydrolysis treatment $(1.11 \pm 0.09 \mathrm{mg} \mathrm{TE} / \mathrm{g}$ $\mathrm{DW}, 4.57 \pm 0.25 \mathrm{mg} \mathrm{TE} / \mathrm{g} \mathrm{DW}$, respectively), indicating that acid hydrolysis is more efficient in releasing the bound antioxidants from soybean. This may be caused by the different chemical components of the scavenging activity. The effectiveness of phenolic compounds extracted from plant-based foods as antioxidants is frequently different [19]. It has reported that the antioxidant activity does not always depend on the quantities of the constituents, but their chemical nature, because considerable variances existed in the efficacies of compounds [32]. In addition, kidney bean had the highest antioxidant activity in the two antioxidant tests, which coincides with the above results, that is, kidney bean showed the highest TPC and TFC.

It is well known that food rich in phenolics and flavonoids possess excellent antioxidant properties. In this study, Pearson's correlation coefficient $\left(R^{2}\right)$ was used to analyze the correlation between the phenolic contents (TPC and TFC) and antioxidant activities (ABTS and FRAP) (Table S3). The results showed that TFC was significantly correlated with ABTS $\left(R^{2}=0.929\right)$ and FRAP $\left(R^{2}=0.977\right)$, while TPC was weakly correlated with ABTS $\left(R^{2}=0.414\right)$ and FRAP $\left(R^{2}=0.455\right)$. It can be seen from Table 3 that a high concentration of catechins contributes dramatically to the content of total flavonoids in kidney beans and broad beans, which indicates that flavonoids, especially concentrated catechins, are the main contributors to the reducibility of kidney beans and vicia faba. Meanwhile, these considerable positive correlations indicate that flavonoids are the main contributors to the antioxidant activities of the three legumes, rather than the phenolic compounds. This result is consistent with previous research results that TFC and FRAP in black soybean seed coat extract also had a strong positive correlation [17].

\subsection{The Effect of In Vitro Gastrointestinal Digestion on Bound Phenolics in Three Legumes}

The quantitative results of the bound phenolics after digestion are shown in Table 4. Compared with acid and alkaline hydrolysis treatment, the release of phenolics was extremely low after digestion. For soybean, the total phenolic content released was $2.09 \pm 0.23 \mu \mathrm{g} / \mathrm{g}$ DW, $2.80 \pm 0.54 \mu \mathrm{g} / \mathrm{g} \mathrm{DW}$, and $3.80 \pm 0.36 \mu \mathrm{g} / \mathrm{g}$ DW after simulated oral, gastric, and intestinal digestion, respectively, while for vicia faba it increased to $2.08 \pm 0.40 \mu \mathrm{g} / \mathrm{g}$ DW, $2.8 \pm 0.72 \mu \mathrm{g} / \mathrm{g}$ DW, and $5.05 \pm 0.12 \mu \mathrm{g} / \mathrm{g} \mathrm{DW}$, respectively, and in kidney bean increased to $2.99 \pm 0.23 \mu \mathrm{g} / \mathrm{g}$ DW, $4.40 \pm 0.59 \mu \mathrm{g} / \mathrm{g} \mathrm{DW}$, and $7.75 \pm 0.59 \mu \mathrm{g} / \mathrm{g}$ DW, respectively. More importantly, the content of phenolics produced by intestinal digestion was higher than that produced by oral and stomach digestion in the three legumes. The release of phenolics in the simulated digestion process was about $3.25-14.63 \%$ of the alkaline hydrolysis treatment, and $3.65-16.75 \%$ of the acid hydrolysis treatment, among which simulated intestinal digestion showed a higher ratio $(16.75 \%$ and $14.63 \%$, respectively) than oral and stomach digestion. In addition, the phenolic compounds released during the simulated digestion process were different in the three legumes. As for soybean, isoflavones were the main species of phenolic compounds, whereas phenolic acids and flavanes (catechin) were mainly released in vicia faba and kidney bean. More specifically, daidzein, daidzin, genistin, and glycitein were the main compounds released after simulated digestion in soybeans; p-hydroxybenzoic acid, p-coumaric acid, and catechin were released in vicia faba; and ferulic acid, p-hydroxybenzoic acid, procatechuic acid, and catechin were released in kidney bean. 
Table 4. Quantitative results of the phenolics after digestion by HPLC-ESI-QqQ-MS a.

\begin{tabular}{|c|c|c|c|c|c|c|c|c|c|}
\hline \multirow{2}{*}{ Compounds } & \multicolumn{3}{|c|}{ Soybean $(\mu \mathrm{g} / \mathrm{g} D W)$} & \multicolumn{3}{|c|}{ Vicia Faba $(\mu \mathrm{g} / \mathrm{g}$ DW) } & \multicolumn{3}{|c|}{ Kidney Bean ( $\mu \mathrm{g} / \mathrm{g} D W)$} \\
\hline & Oral & Gastric & Intestinal & Oral & Gastric & Intestinal & Oral & Gastric & Intestinal \\
\hline \multicolumn{10}{|c|}{ Phenolic acids } \\
\hline p-hydroxybenz-oic acid & $0.01 \pm 0.01$ & $0.05 \pm 0.01$ & $0.16 \pm 0.02$ & $0.29 \pm 0.09$ & $0.54 \pm 0.33$ & $0.69 \pm 0.02$ & $0.61 \pm 0.06$ & $0.72 \pm 0.10$ & $1.22 \pm 0.19$ \\
\hline procatechuic acid & $\mathrm{Nd}$ & $\mathrm{Nd}$ & $\mathrm{Nd}$ & $0.16 \pm 0.02$ & $0.22 \pm 0.03$ & $0.10 \pm 0.01$ & $0.40 \pm 0.04$ & $0.78 \pm 0.11$ & $1.28 \pm 0.02$ \\
\hline ferulic acid & $\mathrm{Nd}$ & $\mathrm{Nd}$ & $\mathrm{Nd}$ & nd & nd & nd & $1.08 \pm 0.03$ & $2.49 \pm 0.32$ & $2.09 \pm 0.23$ \\
\hline chlorogenic acid & $0.13 \pm 0.01$ & $0.12 \pm 0.01$ & $0.16 \pm 0.07$ & $0.19 \pm 0.04$ & $0.14 \pm 0.02$ & $0.20 \pm 0.04$ & nd & nd & $0.11 \pm 0.02$ \\
\hline sinapic acid & nd & nd & nd & $\mathrm{Nd}$ & nd & nd & $0.01 \pm 0.01$ & $0.03 \pm 0.01$ & $0.06 \pm 0.01$ \\
\hline gallic aicd & nd & nd & nd & $\mathrm{Nd}$ & nd & nd & nd & nd & nd \\
\hline p-coumaric acid & $0.08 \pm 0.01$ & $0.09 \pm 0.01$ & $0.10 \pm 0.01$ & $0.50 \pm 0.05$ & $0.81 \pm 0.01$ & $0.34 \pm 0.01$ & $0.08 \pm 0.01$ & $0.08 \pm 0.01$ & $0.10 \pm 0.01$ \\
\hline & & & & $\begin{array}{l}\text { Flavonoids } \\
\text { Isoflavones }\end{array}$ & & & & & \\
\hline daidzein & $0.17 \pm 0.05$ & $0.30 \pm 0.12$ & $0.46 \pm 0.04$ & nd & nd & nd & nd & nd & nd \\
\hline daidzin & $0.80 \pm 0.02$ & $0.85 \pm 0.20$ & $1.10 \pm 0.06$ & $0.04 \pm 0.01$ & $0.04 \pm 0.01$ & $0.06 \pm 0.01$ & nd & nd & nd \\
\hline genistin & $0.71 \pm 0.03$ & $0.99 \pm 0.12$ & $1.26 \pm 0.10$ & nd & nd & nd & nd & nd & nd \\
\hline glycitein & $0.08 \pm 0.06$ & $0.31 \pm 0.04$ & $0.44 \pm 0.03$ & nd & nd & nd & nd & nd & nd \\
\hline glycitin & $0.09 \pm 0.02$ & $0.06 \pm 0.01$ & $0.06 \pm 0.01$ & nd & nd & nd & nd & nd & nd \\
\hline rutin & nd & nd & nd & nd & nd & nd & $0.19 \pm 0.04$ & $0.09 \pm 0.01$ & $0.09 \pm 0.01$ \\
\hline vitexin & $0.01 \pm 0.01$ & $0.02 \pm 0.01$ & $0.04 \pm 0.01$ & 0.004 & 0 & 0.009 & nd & nd & nd \\
\hline & & & & Flavanones & & & & & \\
\hline naringenin & $0.01 \pm 0.01$ & $0.01 \pm 0.01$ & $0.02 \pm 0.01$ & $\begin{array}{c}\text { nd } \\
\text { Flavanes }\end{array}$ & nd & nd & nd & nd & nd \\
\hline catechin & nd & nd & nd & $0.90 \pm 0.19$ & $1.05 \pm 0.32$ & $3.66 \pm 0.03$ & $0.62 \pm 0.04$ & $0.21 \pm 0.03$ & $2.67 \pm 0.09$ \\
\hline Total & $2.09 \pm 0.23$ & $2.80 \pm 0.54$ & $3.80 \pm 0.36$ & $2.08 \pm 0.40$ & $2.80 \pm 0.72$ & $5.05 \pm 0.12$ & $2.99 \pm 0.23$ & $4.40 \pm 0.59$ & $7.75 \pm 0.59$ \\
\hline $\begin{array}{l}\text { Percent of acid } \\
\text { hydrolysis/\% }\end{array}$ & 9.22 & 12.34 & 16.75 & 5.04 & 6.79 & 12.25 & 3.65 & 5.36 & 9.45 \\
\hline $\begin{array}{l}\text { Percent of alkaline } \\
\text { hydrolysis/\% }\end{array}$ & 8.04 & 10.78 & 14.63 & 3.25 & 4.38 & 7.9 & 4.04 & 5.95 & 10.48 \\
\hline
\end{tabular}

${ }^{a}$ Results are expressed as mean \pm standard deviation of three replicates. 
During simulated gastrointestinal digestion, enzymes and $\mathrm{pH}$ conditions can promote the hydrolysis of macromolecules [33]. In this study, intestinal digestion was more effective than oral and gastric digestion in releasing bound phenolics, which was attributed to the abundance of hydrolases in the small intestine. Phenolic compounds may be bound to proteins, so they can be released in the enzyme digestion, and become more stable under the $\mathrm{pH}$ conditions [34]. The results may show that some phenolic acids and flavonoids in these three legumes were bound to the proteins, and such bound phenolics were varied in the different kinds of beans.

Moreover, some bound phenolic compounds are mainly covalent binding with cell wall substances, but due to the inability of the cell wall fibrous material to be digested, such bound phenolic compounds are retained in the gastric and small intestine, and finally arrive at the colon to be released through the action of several microorganisms and hydrolytic enzymes. Kroon et al. [35] proposed that over $95 \%$ of ferulic acid in wheat was released by the enzymatic action (esterase and xylanase activity) of the microbial community during the fermentation in the colon. However, the gastric and small intestinal treatments only released a small amount of ferulic acid (2.6\%). Shahidi and Judong [36] showed that phenolic compounds are rarely produced from the food matrix, and are absorbed in the small intestine (5-10\%) as the free form, and the remainder move to the colon (large intestine) where the bound phenolics are released by the gut microbiota.

\subsection{The Effect of Colonic Fermentation by Human Microflora on Bound Phenolics in Three Legumes}

Phenolics in the diet are difficult to absorb in the small intestine and reach the colon, where they are transformed and degraded by microorganisms. Therefore, in vitro colon fermentation of the bound phenolics in three legumes was carried out. The $\mathrm{pH}$ value and the release of bound phenolics of the three legumes during different fermentation times were detected (Table S4, Table 5). Moreover, the compositions of bound phenolics in the three legumes during different fermentations were identified. The total ion chromatogram of phenolic extracts in the three legumes after colonic fermentation is shown in Figure 2. The retention times and $\mathrm{MS}^{2}$ data of identified phenolics in three legumes after colonic fermentation are shown in (Table 6).

Table 5. Total phenolic contents for different times of colonic fermentation (mg GAE/g DW) ${ }^{\mathrm{A}}$.

\begin{tabular}{|c|c|c|c|c|c|c|c|c|}
\hline & $0 \mathrm{~h}$ & $1 \mathrm{~h}$ & $3 \mathrm{~h}$ & $6 \mathrm{~h}$ & $12 \mathrm{~h}$ & $24 \mathrm{~h}$ & $36 \mathrm{~h}$ & $48 \mathrm{~h}$ \\
\hline Soybean & $0^{\mathrm{b}}$ & $1.43 \pm 0.04^{\mathrm{a}}$ & $1.50 \pm 0.19^{a}$ & $1.64 \pm 0.17^{\mathrm{a}}$ & $1.49 \pm 0.24^{a}$ & $1.72 \pm 0.26^{\mathrm{a}}$ & $1.30 \pm 0.15^{a}$ & $1.65 \pm 0.01^{a}$ \\
\hline Vicia faba & $0^{c}$ & $0.85 \pm 0.11^{b}$ & $0.93 \pm 0.25^{b}$ & $0.91 \pm 0.25^{b}$ & $1.16 \pm 0.09 \mathrm{ab}$ & $1.29 \pm 0.28^{a b}$ & $1.48 \pm 0.11^{\mathrm{a}}$ & $1.45 \pm 0.26^{\mathrm{a}}$ \\
\hline $\begin{array}{c}\text { Kidney } \\
\text { bean }\end{array}$ & $0^{\mathrm{c}}$ & $0.73 \pm 0.17^{a}$ & $0.40 \pm 0.12^{b}$ & $0.56 \pm 0.19 \mathrm{ab}$ & $0.59 \pm 0.06^{\mathrm{ab}}$ & $0.79 \pm 0.12^{\mathrm{a}}$ & $0.82 \pm 0.10^{a}$ & $0.81 \pm 0.06^{a}$ \\
\hline
\end{tabular}

${ }^{A}$ Results are expressed as mean \pm standard deviation of three replicates; Values followed by the different letters $(\mathrm{a}, \mathrm{b}, \mathrm{c})$ within the same line are significantly different $(P<0.05)$.

\subsubsection{The Change of $\mathrm{pH}$ During Colonic Fermentation}

Colon microbes have a high catabolic activity. They can degrade the components in legume residues, and then change the acidity and alkalinity of the solution [37]. The changes of $\mathrm{pH}$ in soybean, broad bean, and kidney bean at different fermentation times are shown in Table S4. With the increase of fermentation time, the $\mathrm{pH}$ values of soybean, vicia faba, and kidney bean were decreased. Interestingly, the $\mathrm{pH}$ value of soybean decreased slowly, and the change range within 48 hours was only 0.64 , which was significantly lower than the downtrend of vicia faba (2.91) and kidney bean (2.69). It may be that soybean is rich in fat and protein, while vicia faba and kidney bean belong to the high starch beans, and their starch content is significantly higher than soybean. Starch fermentation produces lactic acid and other substances, which can cause the $\mathrm{pH}$ of vicia faba and kidney bean to decrease during colon fermentation. Moreover, the $\mathrm{pH}$ of vicia faba and kidney bean decreased slowly in $1 \mathrm{~h}$, but decreased significantly in 1-12 $\mathrm{h}$, then tended to be stable after $36 \mathrm{~h}$. Many studies have suggested that only a few gut microbiota (e.g., Lactobacillus sp., Escherichia coli, Bacteroides sp., Bifidobacterium sp.,) can biotransform dietary polyphenols, and the fermentation products are mainly phenylpropionic acid and phenylacetic acid 
or its hydroxyl derivatives [38,39], which can decrease the $\mathrm{pH}$ of the gut, and therefore the effect of microorganisms in the early stage of food reaching the colon is more significant.

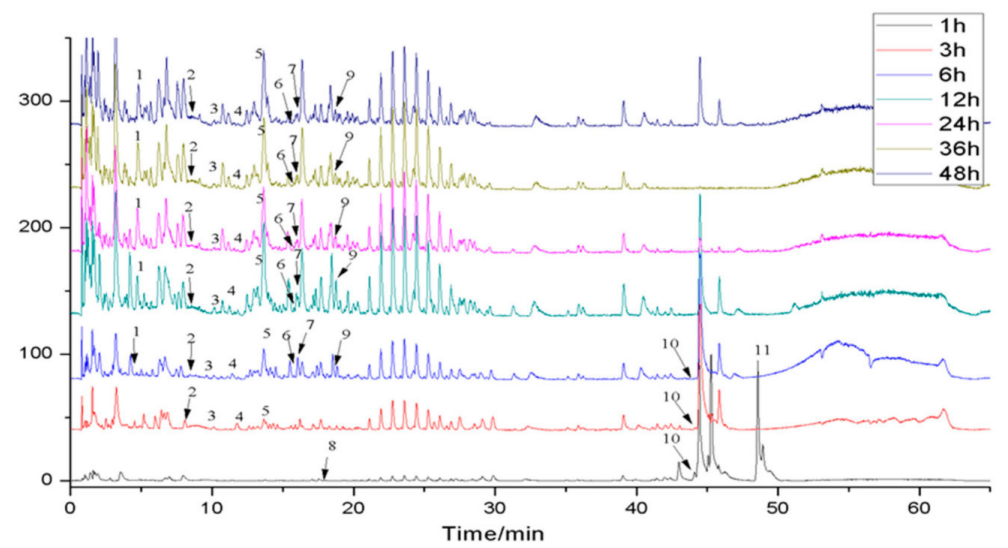

(a) Kidney bean

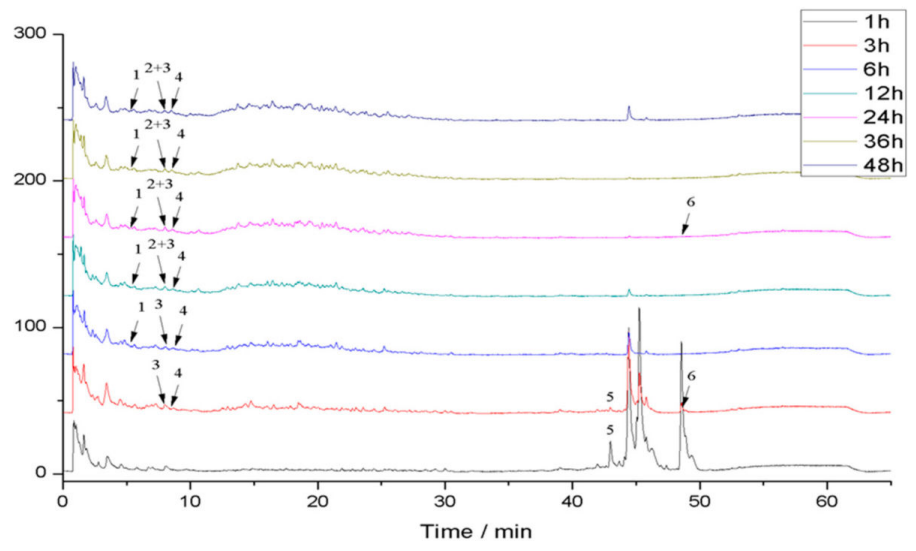

(b) Vicia faba

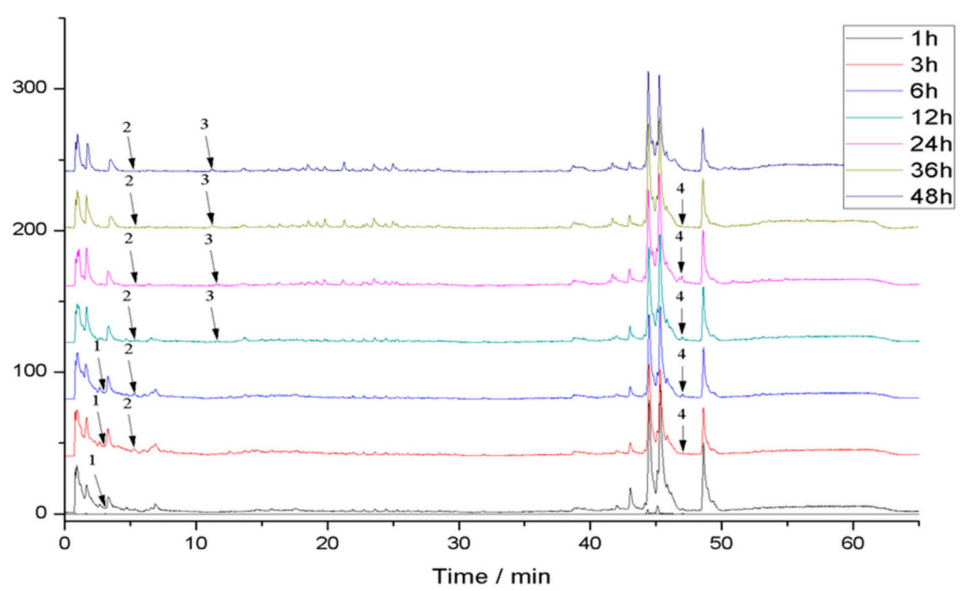

(c) Soybean

Figure 2. Total ion chromatogram (TIC) of phenolic extracts in legumes after colonic fermentation. 
Table 6. Characterization of compounds during colonic fermentation by UPLC-ESI-QTOF-MS2.

\begin{tabular}{|c|c|c|c|c|c|c|c|c|c|}
\hline & \multirow{2}{*}{$\begin{array}{c}t_{R} / \\
(\min )\end{array}$} & \multirow{2}{*}{ Formula } & \multirow{2}{*}[\mathbf{M}-\mathbf{H}]{$^{-}(m / z)$} & \multirow{2}{*}{ Fragment Ions $(\mathrm{m} / \mathrm{z})$} & \multirow{2}{*}{ Identification } & \multicolumn{4}{|c|}{ Fermentation Time } \\
\hline & & & & & & $1 \mathrm{~h}$ & $3 \mathrm{~h}$ & $6 \mathrm{~h}$ & $12 \mathrm{~h}$ \\
\hline \multicolumn{10}{|c|}{ Kidney Bean } \\
\hline 1 & 4.635 & $\mathrm{C}_{14} \mathrm{H}_{12} \mathrm{O}_{5}$ & 259.1302 & 241.1191,197.1293,171.1476 & 2,6,4'-trihydroxy-4-methoxybenzophenone ${ }^{b}$ & & & $\sqrt{ }$ & $\sqrt{ }$ \\
\hline 2 & 8.305 & $\mathrm{C}_{14} \mathrm{H}_{14} \mathrm{O}_{3}$ & 229.154 & 185.1658 & (Iso)pentenyl-7-hydroxy-coumarin ${ }^{\mathrm{b}}$ & & $\sqrt{ }$ & $\sqrt{ }$ & $\sqrt{ }$ \\
\hline 3 & 10.142 & $\mathrm{C}_{7} \mathrm{H}_{6} \mathrm{O}_{3}$ & 137.0578 & $95.0489,122.0349$ & Hydroxybenzoic acid abc & & $\sqrt{ }$ & $\sqrt{ }$ & $\sqrt{ }$ \\
\hline 4 & 11.852 & $\mathrm{C}_{13} \mathrm{H}_{7} \mathrm{O}_{5}$ & 243.1684 & 199.179 & Xanthotoxol acette ${ }^{b}$ & & $\sqrt{ }$ & $\sqrt{ }$ & $\sqrt{ }$ \\
\hline 5 & 13.82 & $\mathrm{C}_{13} \mathrm{H}_{7} \mathrm{O}_{5}$ & 243.1651 & $199.1807,182.1586$ & Xanthotoxol acette ${ }^{b}$ & & $\sqrt{ }$ & $\sqrt{ }$ & $\sqrt{ }$ \\
\hline 6 & 15.591 & $\mathrm{C}_{14} \mathrm{H}_{12} \mathrm{O}_{4}$ & 243.17 & & Benzophenone ${ }^{\mathrm{b}}$ & & & $\sqrt{ }$ & $\sqrt{ }$ \\
\hline 7 & 16.069 & & 327.1298 & 291.1036 & Brevifolin-carboxylic acid derivative ${ }^{a b}$ & & & $\sqrt{ }$ & $\sqrt{ }$ \\
\hline 8 & 17.986 & $\mathrm{C}_{10} \mathrm{H}_{12} \mathrm{O}_{4}$ & 195.0653 & & Acetosyringone ${ }^{b}$ & $\sqrt{ }$ & & & \\
\hline 9 & 18.832 & $\mathrm{C}_{17} \mathrm{H}_{25} \mathrm{O}_{3}$ & 277.1516 & & Decyloxy benzoic acid ${ }^{b}$ & & & $\sqrt{ }$ & $\sqrt{ }$ \\
\hline 10 & 44.099 & & 391.2823 & 223.3473 & Sinapic acid derivative ${ }^{a b}$ & $\sqrt{ }$ & $\sqrt{ }$ & $\sqrt{ }$ & \\
\hline 11 & 48.601 & & 391.2902 & 343.2653 & $\begin{array}{l}\text { 6-Hydroxy kaempferol 3,6,7-trimethyl } \\
\text { ether derivative }\end{array}$ & $\sqrt{ }$ & & & \\
\hline \multicolumn{10}{|c|}{ Vicia Faba } \\
\hline 1 & 5.277 & $\mathrm{C}_{9} \mathrm{H}_{10} \mathrm{O}_{2}$ & 149.0689 & & 4-ethylbenzoic acid ${ }^{b}$ & & & $\sqrt{ }$ & $\sqrt{ }$ \\
\hline 2 & 7.996 & $\mathrm{C}_{9} \mathrm{H}_{8} \mathrm{O}_{3}$ & 163.1728 & & Hydroxyphenyl acrylate $b$ & & & & $\sqrt{ }$ \\
\hline 3 & 8.001 & $\mathrm{C}_{9} \mathrm{H}_{10} \mathrm{O}_{4}$ & 181.0481 & $163.0412,131.1925$ & Dihydroxybenzene propionic acid ${ }^{b}$ & & $\sqrt{ }$ & $\sqrt{ }$ & $\sqrt{ }$ \\
\hline 4 & 8.099 & $\mathrm{C}_{14} \mathrm{H}_{14} \mathrm{O}_{3}$ & 229.1533 & 185.1668 & (Iso)pentenyl-7-hydroxy-coumarin ${ }^{b}$ & & $\sqrt{ }$ & $\sqrt{ }$ & $\sqrt{ }$ \\
\hline 5 & 43.049 & & 391.2808 & 223.3473 & Sinapic acid derivative ${ }^{a b}$ & $\sqrt{ }$ & $\sqrt{ }$ & & \\
\hline 6 & 48.614 & & 391.2812 & 343.2653 & $\begin{array}{l}\text { 6-Hydroxycalanol 3,6,7-trimethyl } \\
\text { ether derivative }^{\text {b }}\end{array}$ & & $\sqrt{ }$ & & \\
\hline \multicolumn{10}{|c|}{ 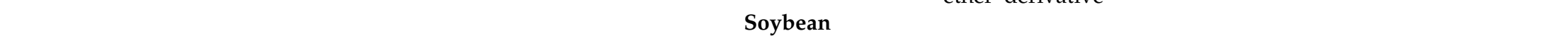 } \\
\hline 1 & 3.079 & $\mathrm{C}_{16} \mathrm{H}_{10} \mathrm{O}$ & 217.1187 & $187.1087,130.0877$ & 1-hydroxyindole ${ }^{b}$ & $\sqrt{ }$ & $\sqrt{ }$ & $\sqrt{ }$ & \\
\hline 2 & 8.103 & $\mathrm{C}_{14} \mathrm{H}_{14} \mathrm{O}_{3}$ & 229.1593 & 185.0603 & (Iso)pentenyl-7-hydroxy-coumarin ${ }^{b}$ & & $\sqrt{ }$ & $\sqrt{ }$ & $\sqrt{ }$ \\
\hline 3 & 11.612 & $\mathrm{C}_{8} \mathrm{H}_{8} \mathrm{O}_{3}$ & 151.0448 & $113.0778,109.1214$ & Hydroxyphenylacetic acid ${ }^{a b}$ & & & & $\sqrt{ }$ \\
\hline 4 & 47.147 & $\mathrm{C}_{20} \mathrm{H}_{22} \mathrm{O}_{8}$ & 389.266 & $435.2747,226.6650$ & Resveratrol glucoside ${ }^{a b}$ & & $\sqrt{ }$ & $\sqrt{ }$ & $\sqrt{ }$ \\
\hline
\end{tabular}

${ }^{a}$ Compared with the literature. ${ }^{b}$ Compared with MSn data, databases, and/or characteristic UV spectra. ${ }^{c}$ Compared with an authentic standard. 


\subsubsection{Total Phenolic Contents}

The content of polyphenols in soybean, vicia faba, and kidney bean at different times of fermentation are shown in Table 5. As the fermentation process proceeded, the polyphenol content of vicia faba increased and then tended to stabilize (0.85-1.45 mg GAE/g DW). This showed that the bound phenolic substances in the legume residues were continuously released by the action of microorganisms and microbial enzymes. The content of polyphenols in soybeans changed with the increase of fermentation time, but showed an increasing trend as a whole. The content of polyphenols in kidney bean was the lowest at $3 \mathrm{~h}(0.4 \mathrm{mg} \mathrm{GAE} / \mathrm{g} \mathrm{DW})$, showed an increasing trend after $3 \mathrm{~h}$ (from 0.4 to $0.82 \mathrm{mg} \mathrm{GAE} / \mathrm{g} \mathrm{DW}$ ), and tended to be stable after $24 \mathrm{~h}$. This may be because the bound phenols were continuously released by microorganisms and microbial enzymes, but at the same time the free phenols were degraded by the microorganisms, so the phenolic acid content was reduced.

Comparing with the alkaline hydrolysis and in vitro gastrointestinal digestion, the liberative polyphenols of soybean and vicia faba during colonic fermentation were higher, reaching $70.5 \%$ and $80.0 \%$ of the alkaline hydrolysis bound phenolic content, respectively, while the liberative polyphenols of kidney bean were extremely low, and were only $38.6 \%$ of alkaline hydrolysis bound phenolic content. In addition, compared with the soluble polyphenols, the content of phenolic acid released in the fermentation of soybeans, vicia faba, and kidney bean accounted for $84.3 \%, 69.1 \%$, and $28.9 \%$ of the soluble polyphenols (Table S1), respectively. The present results imply that the liberative and absorptive effects in vivo of bound polyphenols in soybean and vicia faba were significantly higher than kidney bean. In this study, it was found that the TPC, TFC, and antioxidant activity of kidney beans, among the three legumes, was the highest, but the bioaccessibility in the gastrointestinal tract was the lowest, which indicates that even if the TPC, TFC, and antioxidant activity measured in vitro are high, it does not mean that there is high bioaccessibility in the human body. Similarly, it indicates that the utilization results of bound phenols in vivo may not be consistent with those in vitro.

At present, in vitro fermentation of phenolic compounds is mostly concentrated on extractable phenolic compounds, and few studies have paid attention to the in vitro colonic fermentation of bound phenolic compounds. The concentration of chlorogenic acid, naringin, and rutin standards significantly decreased under the action of intestinal micro-organisms during in vitro colon fermentation [40]. Dall'Asta et al. [41] carried out in vitro colon fermentation of 16 kinds of food juices rich in polyphenols. It was found that the type of phenolic substances changed significantly, and most of the phenolic substances degraded into other phenolic substances after being fermented by microorganisms. Importantly, this study also showed the same results, that changes in the composition of the compounds during different fermentation times indicated that the bound phenolic components were degraded during fermentation in vitro. Under the action of microorganisms, the bound phenolic compounds released during the fermentation of the colon are absorbed by the body in the form of phenolic prototypes or metabolites.

\subsubsection{Phenolics}

The total ion current chromatogram and preliminary identification of the compounds extracted in the three legume residues at different times of fermentation are shown in Figure 2 and Table 6, respectively. The species of compounds in the extracts changed with different fermentation times. More specifically, 11 species of compounds were identified in kidney bean residue, while 6 species were in vicia faba residue, and 4 species were in soybean residue.

For kidney bean, after comparing with the Metlin online database, compounds 1 ( $t_{\mathrm{R}} 4.635 \mathrm{~min}$, $\mathrm{m} / \mathrm{z} 259), 4\left(\mathrm{t}_{\mathrm{R}} 11.852 \mathrm{~min}, \mathrm{~m} / \mathrm{z} 243\right), 6\left(\mathrm{t}_{\mathrm{R}} 15.591 \mathrm{~min}, \mathrm{~m} / \mathrm{z} 243\right), 8\left(\mathrm{t}_{\mathrm{R}} 17.986 \mathrm{~min}, \mathrm{~m} / \mathrm{z} 195\right)$, and 9 $\left(t_{R} 18.832 \mathrm{~min}, \mathrm{~m} / \mathrm{z} 277\right.$ ) were identified as 2,6,4' -trihydroxy-4-methoxybenzophenone, xanthotoxol acette, benzophenone, acetosyringone, and decyloxy benzoic acid, respectively. Compound 5 $\left(t_{R} 13.820 \mathrm{~min}, \mathrm{~m} / \mathrm{z} 243\right.$ ) showed the same molecular ion and fragment ions at $\mathrm{m} / \mathrm{z} 109$, with compound 4 was also identified as xanthotoxol acette; compound $2\left(t_{R} 8.305 \mathrm{~min}, \mathrm{~m} / \mathrm{z} 229\right)$ and compound $11\left(t_{\mathrm{R}} 48.601 \mathrm{~min}, \mathrm{~m} / \mathrm{z}\right.$ 391) were characterized as (Iso)pentenyl-7-hydroxy-coumarin and 6-hydroxy 
kaempferol 3,6,7-trimethyl ether derivative, respectively, by their fragment ions from loss of $\mathrm{CO}_{2}(44 \mathrm{Da})$. Compound $3\left(\mathrm{t}_{\mathrm{R}} 10.142 \mathrm{~min}\right.$ ) with a molecular ion at $\mathrm{m} / \mathrm{z} 137$ was suggested to be hydroxybenzoic acid according to the literature [42]. Brevifolin carboxylic acid derivative (compound $7, t_{R} 16.069 \mathrm{~min}$ ) displayed $[\mathrm{M}-\mathrm{H}]^{-}$at $\mathrm{m} / \mathrm{z} 327$ along with characteristic fragment ions at $\mathrm{m} / \mathrm{z} 291$; compound 10 $\left(\mathrm{t}_{\mathrm{R}} 44.099 \mathrm{~min}\right.$ ) with $[\mathrm{M}-\mathrm{H}]^{-}$at $\mathrm{m} / \mathrm{z} 391$, with a fragment ion at $\mathrm{m} / \mathrm{z} 223$ (sinapic acid), was classified as a sinapic acid derivative [43].

For vicia faba, (Iso) pentenyl-7-hydroxy-coumarin (compound 4, $\mathrm{t}_{\mathrm{R}} 8.099 \mathrm{~min}$ ), sinapic acid derivative (compound 5, $\mathrm{t}_{\mathrm{R}} 43.049 \mathrm{~min}$ ), and 6-hydroxycalanol 3,6,7-trimethyl ether derivative (compound $6, t_{R} 48.614 \mathrm{~min}$ ) were also found in the vicia faba. Compound $1\left(t_{R} 5.277 \mathrm{~min}, \mathrm{~m} / z\right.$ 149), compound $2\left(t_{R} 7.996 \mathrm{~min}, \mathrm{~m} / \mathrm{z} 163\right)$, and compound $3\left(t_{R} 8.001 \mathrm{~min}, \mathrm{~m} / \mathrm{z} 181\right)$ were identified as 4-ethylbenzoic acid, hydroxyphenyl acrylate, and dihydroxybenzene propionic acid, respectively, by comparing with the Metlin online database.

In soybean, (Iso) pentenyl-7-hydroxy-coumarin (compound 2, $t_{\mathrm{R}} 8.103$ ) was also detected. Based on the Metlin online database comparison, compound $1\left(t_{R} 3.097 \mathrm{~min}, \mathrm{~m} / z\right.$ 217) exhibiting fragment ions at $\mathrm{m} / \mathrm{z} 187\left[\mathrm{M}-\mathrm{H}-\mathrm{CO}_{2}\right]^{-}$and $\mathrm{m} / \mathrm{z} 130\left[\mathrm{M}-\mathrm{H}-\mathrm{C}_{3} \mathrm{H}_{2} \mathrm{O}_{3}\right]^{-}$was characterized as 1-hydroxyindole. Compound 3 ( $\mathrm{t}_{\mathrm{R}} 11.621 \mathrm{~min}$ ) exhibited [M-H]- ions at $\mathrm{m} / \mathrm{z} 151$ and fragments at $\mathrm{m} / \mathrm{z} 113$ and $\mathrm{m} / \mathrm{z} 109$ were suggested as hydroxyphenylacetic acid according to the literature data [37]. Compound $4\left(t_{R}=47.147 \mathrm{~min}\right)$ presented a $[\mathrm{M}-\mathrm{H}]^{-}$at $\mathrm{m} / \mathrm{z} 389$, yielding fragments at $\mathrm{m} / \mathrm{z} 227$ (by loss of 162), suggesting that it could be a resveratrol glucoside [44].

The gut microbiota conducts metabolic reactions to decorate phenolic skeletons, to absorb a range of lower-weight metabolites [45]. The phenols in the fermentation system change with the fermentation time due to microbial enzymes that can make phenolics dehydroxyl, ring fission, and chemical bond breakage, and degrade them to other substances [40]. In this study, sinapic acid derivative and 6-hydroxy kaempferol 3,6,7-trimethyl ether derivative mainly existed in the early stage of fermentation of kidney bean and vicia faba residue, and acetosyringone also existed in the early stage of fermentation of kidney bean residue. This indicated that the released phenolic compounds were being rapidly used or degraded into other metabolites by the gut microbiota. For the components of released by bound polyphenols, hydrolysis and fermentation treatment were different. The bound phenolics released by acid or base hydrolysis were mostly individual phenols in the present study, but fermentation mainly produced phenylpropionic acid, phenylacebic acid, and benzoic acid derivatives (end products), which is consistent with the findings of Rechner A R et al. [40]. Moreover, sinapic acid, coumarin, and kaempferol derivatives, which were found in acid or base hydrolysis, were also present in colonic fermentation. A previous study showed that dietary polyphenols were extensively metabolized to simple phenolics (such as propionic acid and phenylacetic acid derivatives) by the colon, and these compounds can be the biomarkers of colonic metabolism [40]. In this study, phenolic acids such as decyloxy benzoic acid, hydroxybenzoic acid, 4-ethylbenzoic acid, and hydroxyphenylacetic acid were found in colon fermentation. This indicated that phenolic derivatives, such as sinapic acid and kaempferol derivatives, may be degraded to simpler phenolics during colonic fermentation.

\section{Conclusions}

The legumes were rich in phenolic compounds and possessed strong antioxidant activity, among which kidney bean showed the highest flavonoid contents and antioxidant activity, but a lower bioavailability than the other two legumes, indicating that the utilization results of bound phenol in vivo may not be consistent with that in vitro. Meanwhile, we found that the antioxidant activity of the three legumes depends on the flavonoid compounds, but not the phenolic compounds. Our results also showed that alkaline hydrolysis was more effective than acid hydrolysis in the release of the bound phenolics of the three legumes. In addition, the bound phenolics were released in extremely low quantities in the in vitro simulated digestion (in oral, stomach, and small intestine digestion), only $3.25-14.63 \%$ of alkali hydrolysis, but were effectively released from the legume matrix under the action of microorganisms (39.61-82.68\% of alkali hydrolysis). The present study also indicated that 
the products released by chemical hydrolysis and in vitro digestion were different; the main products of chemical hydrolysis are phenolic acids (such as protocatechuic acid), which are metabolized into simple phenolics (such as benzoic acid and phenylacetic acid) after colonic fermentation. The results of this study may help us to better understand the phenolic compounds and their biological utilizations in the soybean, vicia faba, and kidney bean, especially the bound phenolics.

Supplementary Materials: The following are available online at http://www.mdpi.com/2304-8158/9/12/1816/s1, Table S1: Total phenolic contents, total flavonoids contents and antioxidant activities of free phenolics in legumes, Table S2. Characterization of free phenolic constituents of legumes by UPLC-ESI-QTOF-MS2, Table S3. Correlation analysis of phenolic and flavonoid contents and antioxidant activity, Table S4. $\mathrm{pH}$ in different time of colonic fermentation, Table S5. The optimized MS parameters of standard substance in the quantitative analysis.

Author Contributions: Funding acquisition, Z.D. and B.Z.; Conceptualization, L.Z. and W.L.; methodology, W.L. and B.Z.; formal analysis, all the authors; investigation, all the authors; writing: original draft preparation, L.Z., W.L., and B.Z.; writing: revision and editing, all the authors. All authors have read and agreed to the published version of the manuscript.

Funding: This work was supported by National Natural Science Foundation of China (Grant No. 31701564), the Natural Science Foundation of Jiangxi Province (Grant No. 20171BAB214035) and Key R\&D Program of Jiangxi Province (Grant No. 20181BBF60014).

Conflicts of Interest: The authors declare no conflict of interest.

\section{References}

1. Vinson, J.A.; Su, X.; Zubik, L.; Bose, P. Phenol antioxidant quantity and quality in foods: Fruits. J. Agric. Food Chem. 2001, 49, 5315-5321. [CrossRef]

2. Robbins, R.J. Phenolic acids in foods: An overview of analytical methodology. J. Agric. Food Chem. 2003, 51, 2866-2887. [CrossRef] [PubMed]

3. Padayachee, A.; Netzel, G.; Netzel, M.; Day, L.; Zabaras, D.; Mikkelsen, D.; Gidley, M.J. Binding of polyphenols to plant cell wall analogues-part 2: Phenolic acids. Food Chem. 2012, 135, 2287-2292. [CrossRef] [PubMed]

4. Yeo, J.; Shahidi, F. Effect of hydrothermal processing on changes of insoluble-bound phenolics of lentils. J. Funct. Foods 2017, 38, 716-722. [CrossRef]

5. Baskaran, R.; Pullencheri, D.; Somasundaram, R. Characterization of of free, esterified and bound phenolics in custard apple (Annona squamosa L.) fruit pulp by UPLC-ESI-MS/MS. Food Res. Int. 2016, 82, 121-127. [CrossRef]

6. Singh, B.; Singh, J.P.; Kaur, A.; Singh, N. Phenolic composition and antioxidant potential of grain legume seeds: A review. Food Res. Int. 2017, 101, 1-16. [CrossRef]

7. Garcialafuente, A.; Moro, C.; Manchon, N.; Gonzaloruiz, A.; Villares, A.; Guillamon, E.; Rostagno, M.A.; Mateovivaracho, L. In vitro anti-inflammatory activity of phenolic rich extracts from white and red common beans. Food Chem. 2014, 161, 216-223. [CrossRef]

8. Mojica, L.; Meyer, A.; Berhow, M.A.; De Mejia, E.G. Bean cultivars (Phaseolus vulgaris L.) have similar high antioxidant capacity, in vitro inhibition of $\alpha$-amylase and $\alpha$-glucosidase while diverse phenolic composition and concentration. Food Res. Int. 2015, 69, 38-48. [CrossRef]

9. Morenovaldespino, C.A.; Lunavital, D.; Camachoruiz, R.M.; Mojica, L. Bioactive proteins and phytochemicals from legumes: Mechanisms of action preventing obesity and type-2 diabetes. Food Res. Int. 2020, 130, 108905. [CrossRef]

10. Inada, K.O.P.; Silva, T.; Lobo, L.A.; Domingues, R.M.C.P.; Perrone, D.; Monteiro, M. Bioaccessibility of phenolic compounds of jaboticaba (Plinia jaboticaba) peel and seed after simulated gastrointestinal digestion and gut microbiota fermentation. J. Funct. Foods 2020, 67, 103851. [CrossRef]

11. Mosele, J.I.; Macià, A.; Romero, M.P.; Motilva, M.J.; Rubió, L. Application of in vitro gastrointestinal digestion and colonic fermentation models to pomegranate products (juice, pulp and peel extract) to study the stability and catabolism of phenolic compounds. J. Funct. Foods 2015, 14, 529-540. [CrossRef]

12. Padayachee, A.; Netzel, G.; Netzel, M.; Day, L.; Mikkelsen, D.; Gidley, M.J. Lack of release of bound anthocyanins and phenolic acids from carrot plant cell walls and model composites during simulated gastric and small intestinal digestion. Food Funct. 2013, 4, 906. [CrossRef] [PubMed] 
13. Minekus, M.; Alminger, M.; Alvito, P.; Ballance, S.; Bohn, T.; Bourlieu, C.; Carriere, F.; Boutrou, R.; Corredig, M.; Dupont, D. A standardised static in vitro digestion method suitable for food-an international consensus. Food Funct. 2014, 5, 1113-1124. [CrossRef] [PubMed]

14. Peng, H.; Li, W.; Li, H.; Deng, Z.; Zhang, B. Extractable and non-extractable bound phenolic compositions and their antioxidant properties in seed coat and cotyledon of black soybean (Glycinemax (L.) merr). J. Funct. Foods 2017, 32, 296-312. [CrossRef]

15. Kim, K.H.; Rong, T.; Yang, R.; Cui, S.W. Phenolic acid profiles and antioxidant activities of wheat bran extracts and the effect of hydrolysis conditions. Food Chem. 2006, 95, 466-473. [CrossRef]

16. Zhang, X.; Zhu, X.; Sun, Y.; Hu, B.; Sun, Y.; Jabbar, S.; Zeng, X. Fermentation in vitro of EGCG, GCG, and EGCG3\Me isolated from Oolong tea by human intestinal microbiota. Food Res. Int. 2013, 54, 1589-1595. [CrossRef]

17. Zhang, B.; Deng, Z.; Ramdath, D.D.; Tang, Y.; Chen, P.X.; Liu, R.; Liu, Q.; Tsao, R. Phenolic profiles of 20 canadian lentil cultivars and their contribution to antioxidant activity and inhibitory effects on $\alpha$-glucosidase and pancreatic lipase. Food Chem. 2015, 172, 862-872. [CrossRef]

18. Sun, Y.; Qin, Y.; Li, H.; Peng, H.; Chen, H.; Xie, H.-r.; Deng, Z. Rapid characterization of chemical constituents in radix tetrastigma, a functional herbal mixture, before and after metabolism and their antioxidant/antiproliferative activities. J. Funct. Foods 2015, 18, 300-318. [CrossRef]

19. Chen, G.L.; Zhang, X.; Chen, S.G.; Han, M.D.; Gao, Y.Q. Antioxidant activities and contents of free, esterified and insoluble-bound phenolics in 14 subtropical fruit leaves collected from the south of china. J. Funct. Foods 2017, 30, 290-302. [CrossRef]

20. Wang, Y.K.; Zhang, X.; Chen, G.L.; Yu, J.; Yang, L.Q.; Gao, Y.Q. Antioxidant property and their free, soluble conjugate and insoluble-bound phenolic contents in selected beans. J. Funct. Foods 2016, 24, 359-372. [CrossRef]

21. Kim, J.S. Antioxidant activities of selected berries and their free, esterified, and insoluble-bound phenolic acid contents. Prev. Nutr. Food Sci. 2018, 23, 35-45. [CrossRef] [PubMed]

22. Kan, L.; Nie, S.; Hu, J.; Liu, Z.; Xie, M. Antioxidant activities and anthocyanins composition of seed coats from twenty-six kidney bean cultivars. J. Funct. Foods 2016, 26, 622-631. [CrossRef]

23. Yasir, M.; Sultana, B.; Amicucci, M. Biological activities of phenolic compounds extracted from amaranthaceae plants and their LC/ESI-MS/MS profiling. J. Funct. Foods 2016, 26, 645-656. [CrossRef]

24. Aguilera, Y.; Dueñas, M.; Estrella, I.; Hernandez, T.; Benitez, V.; Esteban, R.M.; Martin-Cabrejas, M.A. Evaluation of phenolic profile and antioxidant properties of pardina lentil as affected by industrial dehydration. J. Agric. Food Chem. 2010, 58, 10101-10108. [CrossRef]

25. Różalska, S.; Szewczyk, R.; Długoński, J. Biodegradation of 4-n-nonylphenol by the non-ligninolytic filamentous fungus gliocephalotrichum simplex: A proposal of a metabolic pathway. J. Hazard. Mater. 2010, 180, 323-331. [CrossRef]

26. López-Cobo, A.; Gómez-Caravaca, A.M.; Pasini, F.; Caboni, M.F.; Segura-Carretero, A.; Fernández-Gutiérrez, A. HPLC-DAD-ESI-QTOF-MS and HPLC-FLD-MS as valuable tools for the determination of phenolic and other polar compounds in the edible part and by-products of avocado. LWT Food Sci. Technol. 2016, 73, 505-513. [CrossRef]

27. Rajauria, G.; Foley, B.; Abu-Ghannam, N. Identification and characterization of phenolic antioxidant compounds from brown irish seaweed himanthalia elongata using LC-DAD-ESI-MS/MS. Innov. Food Sci. Emerg. Technol. 2016, 37, 261-268. [CrossRef]

28. Zheng, Z.; Li, S.; Zhong, Y.; Zhan, R.; Yan, Y.; Pan, H.; Yan, P. UPLC-QTOF-MS identification of the chemical constituents in rat plasma and urine after oral administration of rubia cordifolia L. Extract. Molecules 2017, 22, 1327. [CrossRef]

29. Prommajak, T.; Sang, M.K.; Pan, C.H.; Sang, M.K.; Rattanapanone, N. Identification of antioxidants in young mango leaves by LC-ABTS and LC-MS. Chiang Mai Univ. J. Nat. Sci. 2014, 13, 317-330. [CrossRef]

30. Acosta-Estrada, B.A.; Gutierrez-Uribe, J.A.; Serna-Saldivar, S.O. Bound phenolics in foods, a review. Food Chem. 2014, 152, 46-55. [CrossRef]

31. Chiremba, C.; Rooney, L.W.; Beta, T. Microwave-assisted extraction of bound phenolic acids in bran and flour fractions from sorghum and maize cultivars varying in hardness. J. Agric. Food Chem. 2012, 60, 4735-4742. [CrossRef] 
32. Martins, N.; Barros, L.; Ferreira, I.C. In vivo antioxidant activity of phenolic compounds: Facts and gaps. Trends Food Sci. Technol. 2016, 48, 1-12. [CrossRef]

33. Podsędek, A.; Redzynia, M.; Klewicka, E.; Koziołkiewicz, M. Matrix effects on the stability and antioxidant activity of red cabbage anthocyanins under simulated gastrointestinal digestion. BioMed Res. Int. 2014, 2014, 365738. [CrossRef] [PubMed]

34. Hachibamba, T.; Dykes, L.; Awika, J.; Minnaar, A.; Duodu, K.G. Effect of simulated gastrointestinal digestion on phenolic composition and antioxidant capacity of cooked cowpea (vigna unguiculata) varieties. Int. J. Food Sci. Technol. 2013, 48, 2638-2649. [CrossRef]

35. Kroon, P.A.; Faulds, C.B.; Ryden, P.; Robertson, J.A.; Williamson, G. Release of covalently bound ferulic acid from fiber in the human colon. J. Agric. Food Chem. 1997, 45, 661-667. [CrossRef]

36. Fereidoon, S.; Judong, Y. Insoluble-bound phenolics in food. Molecules 2016, 21, 1216.

37. Mosele, J.I.; Macia, A.; Romero, M.P.; Motilva, M.-J. Stability and metabolism of arbutus unedo bioactive compounds (phenolics and antioxidants) under in vitro digestion and colonic fermentation. Food Chem. 2016, 201, 120-130. [CrossRef] [PubMed]

38. Cardona, F.; Andrés-Lacueva, C.; Tulipani, S.; Tinahones, F.J.; Queipo-Ortuño, M.I. Benefits of polyphenols on gut microbiota and implications in human health. J. Nutr. Biochem. 2013, 24, 1415-1422. [CrossRef] [PubMed]

39. Filesi, C.; Benedetto, R.D. Polyphenols, dietary sources and bioavailability. Ann. Ist Super Sanita 2007, 43, 348-361.

40. Rechner, A.R.; Smith, M.A.; Kuhnle, G.; Gibson, G.R.; Debnam, E.S.; Srai, S.K.S.; Moore, K.P.; Rice-Evans, C.A. Colonic metabolism of dietary polyphenols: Influence of structure on microbial fermentation products. Free Radical Biol. Med. 2004, 36, 212-225. [CrossRef]

41. Dall'Asta, M.; Calani, L.; Tedeschi, M.; Jechiu, L.; Brighenti, F.; Del Rio, D. Identification of microbial metabolites derived from in vitro fecal fermentation of different polyphenolic food sources. Nutrition 2012, 28, 197-203. [CrossRef]

42. Brosnan, B.; Coffey, A.; Arendt, E.K.; Furey, A. Rapid identification, by use of the LTQ Orbitrap hybrid FT mass spectrometer, of antifungal compounds produced by lactic acid bacteria. Anal. Bioanal. Chem. 2012, 403, 2983-2995. [CrossRef] [PubMed]

43. Spínola, V.; Pinto, J.; Castilho, P.C. Identification and quantification of phenolic compounds of selected fruits from madeira island by HPLC-DAD-ESI-MS and screening for their antioxidant activity. Food Chem. 2015, 173, 14-30. [CrossRef] [PubMed]

44. Rotches-Ribalta, M.; Andres-Lacueva, C.; Estruch, R.; Escribano, E.; Urpi-Sarda, M. Pharmacokinetics of resveratrol metabolic profile in healthy humans after moderate consumption of red wine and grape extract tablets. Pharmacol. Res. 2012, 66, 375-382. [CrossRef] [PubMed]

45. Aura, A.-M. Microbial metabolism of dietary phenolic compounds in the colon. Phytochem. Rev. 2008, 7, 407-429. [CrossRef]

Publisher's Note: MDPI stays neutral with regard to jurisdictional claims in published maps and institutional affiliations.

(C) 2020 by the authors. Licensee MDPI, Basel, Switzerland. This article is an open access article distributed under the terms and conditions of the Creative Commons Attribution (CC BY) license (http://creativecommons.org/licenses/by/4.0/). 